\title{
Optimal Control Approaches for Analysis of Energy Use Minimization of Hybrid Ground-Coupled Heat Pump Systems
}

\author{
Ercan Atam, Dieter Patteeuw, Stefan P. Antonov, and Lieve Helsen
}

\begin{abstract}
In this paper, we present a prediction-based dynamic programming (DP) control approach, a nonlinear model predictive control (NMPC) approach, and a linear optimal control (LOC) approach to analyze the minimization of the total energy use of a hybrid ground-coupled heat pump (hp) system (incorporating a ground-coupled hp, a gas boiler, a passive cooler, and an active chiller) under operational constraints. A large-scale emulator model (based on finite-volume method and the equivalent-diameter approach) is used for the borehole system and for the assessment of different control algorithms. A nonlinear autoregressive exogenous model is identified from the inputoutput data generated by the emulator model to be used in a DP-based controller. Since DP is a global optimal control method, it was used as a reference for performance assessment. Next, a state-space reduced-order control-oriented model with a larger sampling time is obtained from the emulator model using the so-called proper orthogonal decomposition model reduction technique. This model is used in an NMPC algorithm to see how much NMPC is suboptimal with respect to the DP in terms of annual energy use minimization. Finally, a series of LOCs based on constant hp coefficients of performance is tested to see how much the system performance deteriorates. The control algorithms are used for the satisfaction of heating-cooling demands of three types of buildings: 1) heating dominated; 2) cooling dominated; and 3 ) thermally balanced. The effects of constraining thermal buildup/depletion of ground, variable electricity prices, and marginal violation of thermal comfort on the performance of the different controllers applied are also separately analyzed.
\end{abstract}

Index Terms-Borefield, dynamic programming (DP), hybrid ground-coupled heat pumps, nonlinear model predictive control (NMPC), optimal control.

Manuscript received January 26, 2015; accepted May 17, 2015. Manuscript received in final form May 28, 2015. The work of E. Atam was supported in part by the Agency for Innovation by Science and Technology in Flanders, and in part by the Scientific and Technical Center for the Building Industry for the post-doctoral research work through the VIS SMART GEOTHERM Project. The work of D. Patteeuw was supported by the Katholieke Universiteit Leuven, Leuven, Belgium, within the framework of his Ph.D. through the GOA Project entitled Fundamental Study of a Greenhouse Gas Emission-Free Energy System. The work of S. P. Antonov was supported by the Research Foundation Flanders within the framework of his Ph.D. through the Project entitled Black-Box Model Based Predictive Control of Ground-Coupled Heat Pump Systems. Recommended by Associate Editor D. Vrabie.

E. Atam, D. Patteeuw, and S. P. Antonov are with the Department of Mechanical Engineering, Katholieke Universiteit Leuven, Leuven 3001, Belgium (e-mail: ercan.atam@kuleuven.be; dieter.patteeuw@kuleuven.be; stefan.antonov@kuleuven.be).

L. Helsen is with the Department of Mechanical Engineering, Katholieke Universiteit Leuven, Leuven 3001, Belgium, and also with EnergyVille, Genk 3600, Belgium (e-mail: lieve.helsen@kuleuven.be).

Color versions of one or more of the figures in this paper are available online at http://ieeexplore.ieee.org.

Digital Object Identifier 10.1109/TCST.2015.2445851

\section{NOMENCLATURE}

$c_{e} \quad$ Electricity price.

$c_{g} \quad$ Gas price.

$J \quad$ Total cost.

$N_{c} \quad$ Control horizon.

$N_{p} \quad$ Prediction horizon.

$t_{s} \quad$ Sampling period.

$\dot{P}_{\text {ch }} \quad$ Electrical power used by chiller.

$\dot{P}_{\mathrm{gb}} \quad$ Electrical power used by gas boiler.

$\dot{P}_{\mathrm{hp}} \quad$ Electrical power used by heat pump.

$\dot{P}_{\mathrm{pc}} \quad$ Electrical power used by passive cooler.

$\dot{Q}_{c} \quad$ Cooling load demand.

$\dot{Q}_{\text {ch }} \quad$ Thermal power extracted from the building through active cooling.

$\dot{Q}_{\mathrm{gb}} \quad$ Thermal power supplied to the building by the gas boiler.

$\dot{Q}_{h} \quad$ Heating load demand.

$\dot{Q}_{\mathrm{hp}} \quad$ Thermal power supplied to the building by the heat pump.

$\dot{Q}_{\text {inj }} \quad$ Heat injected to ground.

$\dot{Q}_{\text {net }} \quad$ Net thermal power injected to ground.

$\dot{Q}_{\mathrm{pc}} \quad$ Thermal power extracted from the building through passive cooling.

$T_{a} \quad$ Ambient air temperature.

$T_{f} \quad$ Borehole circulating fluid mean temperature.

$T_{\mathrm{sw}, h} \quad$ Supply water temperature for heating.

$T_{\mathrm{sw}, c} \quad$ Supply water supply temperature for cooling.

$\eta_{\mathrm{gb}} \quad$ Gas boiler efficiency.

bal Balanced.

bh borehole.

cdom Cooling dominated.

ch Chiller.

E Electricity.

$f \quad$ Fluid.

gb Gas boiler.

hdom Heating dominated.

hp Heat pump.

marg. Margin.

pc Passive cooler.

A Annual.

B Building.

COP Coefficient of performance.

$\mathrm{D} / \mathrm{N} \quad$ Day night.

DP Dynamic programming.

GCHP Ground-coupled heat pump. 


$\begin{array}{ll}\text { HyGCHP } & \text { Hybrid ground-coupled heat pump. } \\ \text { LOC } & \text { Linear optimal control. } \\ \text { MPC } & \text { Model predictive control. } \\ \text { NARX } & \text { Nonlinear autoregressive with exogenous input. } \\ \text { NMPC } & \text { Nonlinear model predictive control. } \\ \text { POD } & \text { Proper orthogonal decomposition. } \\ \text { RC } & \text { Relative change. } \\ \text { ROM } & \text { Reduced-order model. } \\ \text { SS } & \text { State space. } \\ \text { TBUD } & \text { Thermal buildup/depletion. }\end{array}$

\section{INTRODUCTION}

$\mathbf{G}$ LOBAL warming and worries about reliable future energy supply have arisen a substantial interest in clean and renewable energy. Especially, last two decades have witnessed a substantial increase in geothermal energy use through GCHPs [1]-[8] and GCHP systems with supplementary devices (HyGCHPs) [9]-[16]. Based on the fact that buildings account for approximately $40 \%$ of total energy use in Europe [18] and similar percentages in the rest of the world, development of energy-efficient buildings and heating and cooling technologies together with appropriate control strategies is required. GCHP systems combined with low-exergy heat emission systems have the potential to reduce the primary energy use of space heating and cooling by $70 \%$ [19] compared with conventional heating and cooling systems. For GCHP systems with vertical borehole heat exchangers, however, the large investment cost of the borefield represents a major bottleneck. This explains the trend toward compact hybrid GCHP systems that combine smaller boreholes with supplementary heating or cooling devices such as gas-fired boilers, passive coolers, and chillers. Although the design of a compact HyGCHP system is often driven by cost considerations to limit the drilling cost without compromising thermal comfort in the building, sometimes other reasons may also lead to HyGCHP systems, such as limited drilling area for boreholes and/or the specific ground characteristics.

Under normal conditions, logically a long-term cost-optimal operation boils down to maximizing the use of the heat pump (hp) and passive cooling in covering the heating and cooling loads within certain temperature constraints for the circulating calorimetric fluid. Although we have this kind of a qualitative nature of optimal control policy in our mind, determination of quantitative values of optimal load sharing between components requires an intelligent computational strategy when multiple operational constraints have to be taken into account. In addition, the possible variability of electricity price should be considered.

In the literature, many strategies toward an intelligent control strategy are available. The first category of studies, for example, [12] and [13], proposed rule-based control strategies coupled with a set of parameters to optimize the system performance using a simulation-based optimization method. The main drawbacks of these approaches are as follows.

1) They suffer from a mathematical model-based optimization/control. Hence, rule-based control methods may be very suboptimal strategies in general.
2) The number of variables to be optimized in a rule-based control is restricted most of the time since few parameters are chosen to characterize the optimal control performance.

In optimal control of HyGCHP systems, the most prominent constraint is the lower-upper temperature bound on the circulating fluid mean temperature. The lower temperature bound is typically defined by the fluid freezing temperature, and the upper temperature bound is defined by constraints on the supply water temperature for direct cooling at building level. Optimal borefield operation thus requires modulation of the borefield heat injection or extraction power to keep the fluid temperature within this range. When maximizing the share of hp or passive cooling in covering the heating and cooling loads, the fluid temperature is, respectively, at the lower or upper bound. Current rule-based control strategies fail to get optimal system operation within (but close to) these physical temperature bound constraints. When operating near the temperature constraints, they often result in an ON-OFF cycling, which is detrimental for both the system energy performance and installation lifetime.

The second category of studies, for example, [14] and [17], in contrast to rule-based approaches, uses mathematical model-based control methods that allow for a better control. However, they are based on simplifications and/or unrealistic assumptions introduced during the controller design. For example, De Ridder et al. [14] applied a DP-based optimal control method. DP is a powerful method since it is a closed-loop global optimal control algorithm. However, the model used in [14] for DP is a very simple first-order model for the ground mean temperature. The chosen control time step for the system is one week, which is very long since typical control actions for buildings may require control time steps in order of minutes or hours. Moreover, the realization of the designed controller requires the measurement of underground field temperature, for which measurement may be either difficult or inaccurate. As a result, the approach in [14] involves both high-level modeling simplifications and a hard-to-realize implementation. Similar to [17], an LOC method is applied. The simplification made in this paper is that the hp COP is taken to be constant without a formal justification. The models used for control and emulator are the same, which eliminates the impact of model mismatch and therefore limits the generality of the approach followed in [17].

In this paper, we present three contributions to both control-oriented modeling and control of HyGCHPs, alleviating the simplifications mentioned above and designing/ validating/analyzing different controller approaches under more realistic assumptions. These contributions are as follows.

As a first contribution, we identify a prediction-oriented low-order (with respect to the number of regressors) neural network NARX model using data from an emulator borehole thermal model for the prediction of circulating fluid mean temperature. The emulator model is a large-scale finitevolume-based numerical borehole thermal model. A low-order NARX is capable of better predictions compared with linear 
models of the same order. Here, since the output variable is the fluid mean temperature, which is the average of inlet and outlet fluid temperatures, it is a measurable quantity in contrast to the mean borefield temperature in [14]. Since the NARX model is a nonlinear model, it is a more sophisticated model aimed at obtaining more accurate predictions and, hence, a better controller design possibility. NARX was used to design a DP-based controller. DP is a closed-loop global optimal control method (global optimal up to the approximations due to input-state gridding and interpolations). The DP control results were used as reference values to compare the performance of other control approaches.

The second contribution is the derivation and use of a control-oriented SS reduced-order model (SS-ROM), which is obtained from the emulator model using the POD model order reduction technique. SS-ROM is used to design a nonlinear model predictive controller to minimize the energy use over the prediction horizon. Since the model is an SS model, an observer is used for the estimation of states. The main objective here was to see how far NMPC-based energy minimization deviates from the global optimal DP control results.

The sampling periods of both NARX and SS-ROM and the control time steps of the designed controllers are $4 \mathrm{~h}$, in contrast to the very long control time step of one week in [14]. As a result, the DP and NMPC controllers based on, respectively, NARX and SS-ROM, are substantially more realistic in terms of control time step. COP values were also taken as functions of source-sink temperatures in both control methods.

The third contribution is the comparison of the results with the reference DP-based control results when a series of linear optimal controllers (LOCs) is designed based on constant hp COP values in the control model. In this way, a formal simulation-based assessment for the performance of linear controllers is provided since the results are compared with the DP results and since all controllers are tested on the emulator model.

In the performance analysis of all the control methods, three types of buildings, heating dominated, cooling dominated, and thermally balanced, are considered to make the analysis results more general. The performance of the control algorithms is analyzed for four cases: 1) without considering TBUD of the ground; 2) constraining the ground to have zero TBUD at the end of the considered period; 3) variable versus $\mathrm{D} / \mathrm{N}$ electricity prices; and 4) allowing some violation margin in the satisfaction of heat-cold loads.

This paper is structured as follows. In Section II, the HyGCHP system and the control problem are described in detail. In Section III, the emulator model of the borehole system to be used both for reduced-order model constructions (NARX and SS-ROM) and for testing the designed controllers is described shortly. Section IV considers control-oriented modeling where an NARX model is identified in Section IV-A and an SS-ROM is constructed in Section IV-B. Prediction-based DP control of the system using NARX model and NMPC of the system using SSROM are elaborated, respectively, in Sections V-A and V-B.

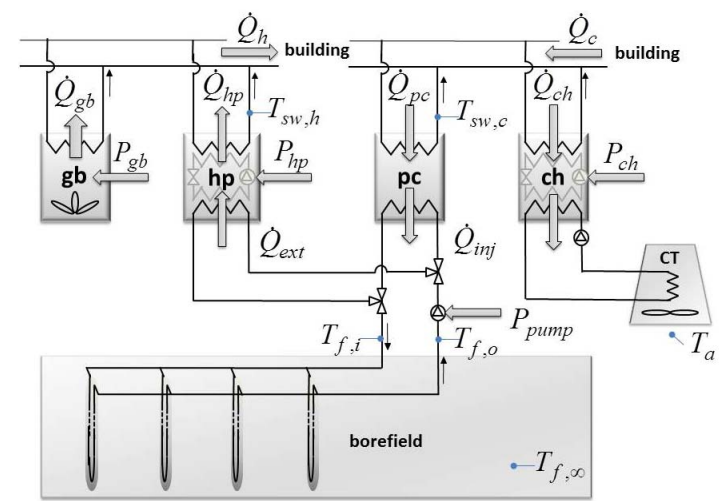

Fig. 1. Schematic of hybrid GCHP system. CT: cooling tower.

Section V-C presents the LOC of the system based on constant hp COP. In the previous sections, TBUD of the ground was not taken into account in the applied control methods. The effect of constraining the ground to have zero TBUD at the end of the control period is analyzed in Section VI. Testing performances of MPC and LOCs under combinations of variable electricity prices and nonzero thermal load violations are considered in Section VII. Lessons learned from this paper are listed in Section VIII. Finally, conclusions of this paper and future work are summarized in Section IX.

\section{System Description And Control Problem}

The hybrid GCHP system is shown in Fig. 1 and consists of a hp, a gas-fired boiler, a passive cooler (pc), and a chiller (ch) (with a cooling tower). It is assumed that the heating demand is provided by the $\mathrm{hp}$ and boiler, and the cooling demand is provided by the pc and ch. The expressions for the efficiency $(\eta)$ and COP of all components shown in Fig. 1 are given by

$\mathrm{COP}_{\mathrm{hp}}=\frac{\dot{Q}_{\mathrm{hp}}}{P_{\mathrm{hp}}}, \eta_{\mathrm{gb}}=\frac{\dot{Q}_{\mathrm{gb}}}{P_{\mathrm{gb}}}, \mathrm{COP}_{\mathrm{pc}}=\frac{\dot{Q}_{\mathrm{pc}}}{P_{\mathrm{pc}}}, \mathrm{COP}_{\mathrm{ch}}=\frac{\dot{Q}_{\mathrm{ch}}}{P_{\mathrm{ch}}}$

where $P_{\mathrm{hp}}$ denotes the electrical power consumption of the $\mathrm{hp}$ compressor and the power consumed by circulation pumps from the borefield side, $P_{\mathrm{gb}}$ denotes the rate of the primary energy use of the gas boiler $(\mathrm{gb}), P_{\mathrm{pc}}$ is the electrical power used for the circulation pumps of the passive cooling installation, and finally, $P_{\mathrm{ch}}$ denotes the electrical power consumption of the ch compressor and the circulation pumps of the cooling tower. The COPs given by the above expressions depend on the temperatures of the source and the emission system, as expressed by

$$
\begin{aligned}
& \mathrm{COP}_{\mathrm{hp}}=f_{\mathrm{hp}}\left(T_{f}, T_{\mathrm{sw}, h}\right) \\
& \mathrm{COP}_{\mathrm{pc}}=f_{\mathrm{pc}}\left(T_{f}, T_{\mathrm{sw}, c}\right) \\
& \mathrm{COP}_{\mathrm{ch}}=f_{\mathrm{ch}}\left(T_{a}, T_{\mathrm{sw}, c}\right)
\end{aligned}
$$

where $T_{\mathrm{sw}, h}$ and $T_{\mathrm{sw}, c}$ represent the supply water temperature for heating and supply water temperature for cooling, respectively. $T_{a}$ is the ambient temperature, and $T_{f}$ is the circulating fluid mean temperature. The gb efficiency, $\eta_{\mathrm{gb}}$, is given a constant value of unity.

Letting $c_{e}(t)$ and $c_{g}$ denote the time-dependent electricity and gas price per kilowatt hour, an optimal control problem 
will be solved to minimize the following cost function for HyGCHP system operation over a time period $\left[t_{0}, t_{f}\right]$ :

$$
\begin{gathered}
J=\int_{t_{0}}^{t_{f}}\left[c_{e}(t)\left(P_{\mathrm{hp}}(t)+P_{\mathrm{pc}}(t)+P_{\mathrm{ch}}(t)\right)+c_{g} P_{\mathrm{gb}}(t)\right] d t \\
\cong \sum_{k=0}^{N-1} t_{s}\left[c _ { e } ( k ) \left(\frac{\dot{Q}_{\mathrm{hp}}(k)}{\operatorname{COP}_{\mathrm{hp}}(k)}+\frac{\dot{Q}_{\mathrm{pc}}(k)}{\operatorname{COP}_{\mathrm{pc}}(k)}\right.\right. \\
\left.\left.\quad+\frac{\dot{Q}_{\mathrm{ch}}(k)}{\operatorname{COP}_{\mathrm{ch}}(k)}\right)+c_{g} \frac{\dot{Q}_{\mathrm{gb}}(k)}{\eta_{\mathrm{gb}}}\right]
\end{gathered}
$$

where $t_{s}$ is the sampling period and $k$ is the sampling instant. The cost function presented by (1) has to be minimized under operational temperature constraints and power constraints. Next, we will discuss these constraints and present their expressions.

\section{A. Heat and Cold Demand Satisfaction}

The building heat and cold demands should be satisfied with some acceptable margins

$$
\begin{aligned}
& \dot{Q}_{h}(k)-\varepsilon_{h-l}(k) \leq \dot{Q}_{\mathrm{hp}}(k)+\dot{Q}_{\mathrm{gb}}(k) \leq \dot{Q}_{h}(k)+\varepsilon_{h-u}(k) \\
& \dot{Q}_{c}(k)-\varepsilon_{c-l}(k) \leq \dot{Q}_{\mathrm{pc}}(k)+\dot{Q}_{\mathrm{ch}}(k) \leq \dot{Q}_{c}(k)+\varepsilon_{c-u}(k)
\end{aligned}
$$

where $\dot{Q}_{h}(k)$ and $\dot{Q}_{c}(k)$ are the building heat and cold demands, respectively, $\varepsilon_{h-l}(k)$ and $\varepsilon_{h-u}(k)$ are the time-dependent lower and upper violation margins for the satisfaction of heat demand, and $\varepsilon_{c-l}(k)$ and $\varepsilon_{c-u}(k)$ are the lower and upper violation margins for the satisfaction of cold demand. Note that the margins are taken to be time dependent to allow different degrees of flexibility over time. During critical demand load periods, these margins can be set very strictly.

\section{B. Circulating Fluid Temperature Bounds}

The cooling of a building requires heat injection to the ground during summer. This increases the ground temperature toward winter, which, in turn, increases $\mathrm{COP}_{\mathrm{hp}}$. However, the ground temperature, which is represented indirectly by $T_{f}$, should be kept below the supply water temperature, $T_{\mathrm{sw}, c}$, for passive cooling of the building. Similarly, heating a building requires heat extraction from the ground. This decreases the ground temperature toward summer, which, in turn, increases $\mathrm{COP}_{\mathrm{pc}}$. However, again the ground temperature represented indirectly by $T_{f}$ should not decrease to a value below freezing point to avoid frost problems. All these require to put lower and upper bounds on $T_{f}$

$$
T_{f-\min }(k)<T_{f}(k)<T_{f-\max }(k) .
$$

$T_{f-\min }$ is set to a small value greater than zero to prevent freezing of the circulating fluid, and $T_{f-\max }$ is set to a value for which passive cooling will be efficient (which depends on climate conditions). In the simulations performed in the next sections, we used $T_{f-\min }=0.5{ }^{\circ} \mathrm{C}$ and $T_{f-\max }=19.5{ }^{\circ} \mathrm{C}$ (based on Belgium climate).

\section{Heat Exchange With the Ground and Bounds on Annual Net Heat Transfer With the Ground}

Heat extraction/injection from/to the ground and the net heat transfer with the ground are given by the following equations:

$$
\begin{aligned}
& \dot{Q}_{\text {ext }}(k)=\frac{\operatorname{COP}_{\mathrm{hp}}(k)-1}{\operatorname{COP}_{\mathrm{hp}}(k)} \dot{Q}_{\mathrm{hp}}(k) \\
& \dot{Q}_{\text {inj }}(k)=\frac{\mathrm{COP}_{\mathrm{pc}}(k)+1}{\operatorname{COP}_{\mathrm{pc}}(k)} \dot{Q}_{\mathrm{pc}}(k) \\
& \dot{Q}_{\text {net }}(k)=\dot{Q}_{\text {inj }}(k)-\dot{Q}_{\text {ext }}(k) .
\end{aligned}
$$

To limit the degree of TBUD in the ground, the following constraint may be put on the net annual heat transfer with the ground:

$$
\left|\sum_{1 \text { year }} \dot{Q}_{\text {net }}\right| \approx 0 .
$$

Note that the constraint in (5) can be used only for optimal control. Since NMPC cannot see the whole control period, it is not applicable for NMPC.

\section{Circulating Fluid Mean Temperature Dynamics}

The model predicting the mean temperature of the circulating fluid will be either an input-output model identified using input-output data from the emulator model in Section IV-A or an SS-ROM derived from the emulator model in Section IV-B. The first model will be used to solve the optimal control problem in Section V-A using DP, and the second one will be used in Section V-B using NMPC.

\section{E. Optimal Control Problem for Total Energy Use Minimization}

The optimal control problem for total energy use minimization will be minimization of (1) over the whole control period subject to the operational/physical constraints given by (2)-(5) plus the dynamics for $T_{f}$ given by (7) in Section V-A.

\section{F. Model Predictive Control Problem for Energy Use Minimization}

In MPC formulation, the control problem for energy use minimization will be minimization of (1) over the prediction horizon subject to the operational/physical constraints given by (2)-(4) plus the dynamics for $T_{f}$ given by (12) in Section V-B. Limits for TBUD in the ground cannot be set here.

\section{Borehole EMUlator Model}

In this section, the borefield, part of the system shown in Fig. 1, is modeled as a single equivalent borehole that is sized according to the specified building loads to be considered in the next sections. This is an approximation neglecting the interaction between different boreholes in a borefield. The equivalent borehole filled with grout is schematically shown in Fig. 2, where an equivalent-diameter approach [4], [10], [21] is used. In the equivalent-diameter approach, the heat transfer from the U-tube is approximated by the heat transfer from a single pipe with a hypothetical diameter through which 


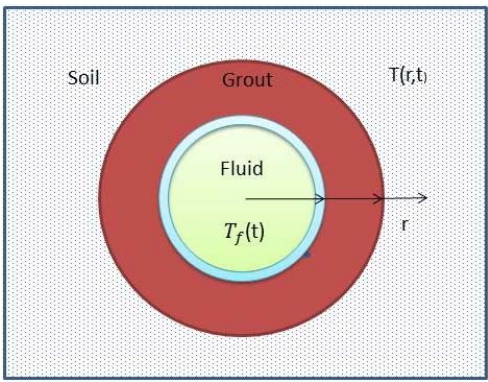

Fig. 2. Equivalent borehole system.

the heat exchanging fluid circulates. The objective is the determination of the circulating fluid mean temperature, $T_{f}(t)$, corresponding to a net (injected-extracted) heat profile per unit length, $u_{\text {net }}=\dot{Q}_{\text {net }}$. The 1-D radial heat conduction equation is considered. Next, the grout and soil regions are divided into thermal nodes and an energy balance for the equivalent borehole is considered. Using the finitevolume technique, the following large-scale dynamic model is obtained:

$$
\begin{aligned}
x_{\mathrm{bh}}(k+1) & =A_{\mathrm{bh}}(p) x_{\mathrm{bh}}(k)+B_{\mathrm{bh}}(p) u_{\mathrm{net}}(k) \\
y(k) & =T_{f}(k)=C_{\mathrm{bh}} x_{\mathrm{bh}}(k)
\end{aligned}
$$

where $x_{\mathrm{bh}}=\left[\begin{array}{lllllll}T_{f} & T_{g_{1}} & \cdots & T_{g_{n g}} & T_{S_{1}} & \cdots & T_{S_{n_{s}}}\end{array}\right]^{T}$. Here, $T_{f}$ is the circulating fluid mean temperature, $\left[\begin{array}{llll}T_{g_{1}} & \cdots & T_{g_{n_{g}}}\end{array}\right]^{T}$ are the grout nodal temperatures, $\left[T_{S_{1}} \cdots T_{S_{n_{\mathrm{s}}}}\right]^{T}$ are the soil (ground) nodal temperatures, $p$ is the known parameter vector including thermal, physical, and other parameters of the system (diffusivities, conductivities, different radii, discretization step sizes, etc.), and $u_{\text {net }}$ is the net heat injected to the ground. A full derivation of (6) can be found in [11].

\section{Control-Oriented Borehole Models}

In this section, we present two models obtained from the borehole emulator model: an input-output model to be used in DP and an SS model to be used in NMPC.

\section{A. Identification of an NARX Prediction Model}

Input-output-based modeling using system identification techniques is a strong alternative modeling approach, which uses only the input and output data and fits an empirical model to it. The type of identification model is strongly depending on the underlying system. The NARX model structure has the general form

$$
\begin{aligned}
& y(k+1) \\
& \quad=\hat{f}_{\text {narx }}\left(y(k), y(k-1), \ldots, u_{\text {net }}(k), u_{\text {net }}(k-1), \ldots\right)
\end{aligned}
$$

where $\hat{f}_{\text {narx }}$ is a nonlinear function. Input arguments to $\hat{f}_{\text {narx }}$ are called model regressors. When the NARX model structure is specified, it is possible to choose among several available nonlinear mapping functions [22], including neural networks, wavelet networks, and so on. Here, we choose a model in two input and two output regressors

$$
y(k+1)=\hat{f}_{\text {narx }}\left(y(k), y(k-1), u_{\text {net }}(k), u_{\text {net }}(k-1)\right)
$$
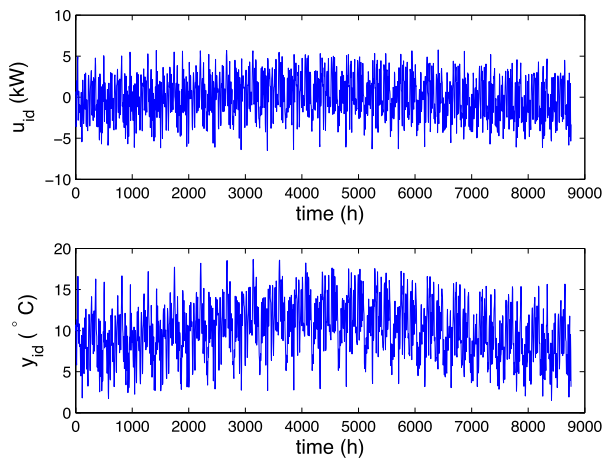

(a)
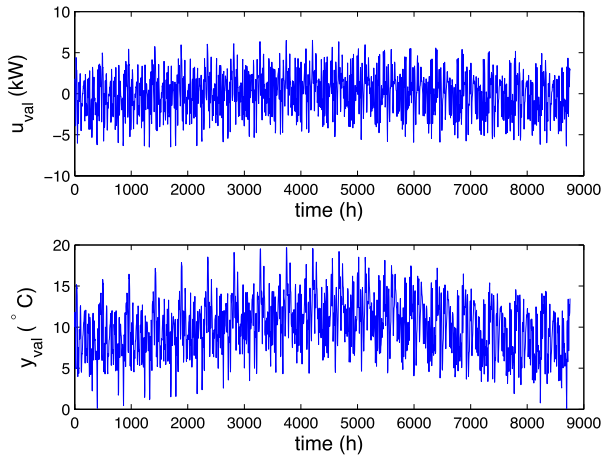

(b)

Fig. 3. (a) Identification data. (b) Validation data.

where $\hat{f}_{\text {narx }}$ is a neural network. Defining the equivalent states $x^{\mathrm{eq}}(k)=\left[\begin{array}{lll}x_{1}^{\mathrm{eq}}(k) & x_{2}^{\mathrm{eq}}(k) & x_{3}^{\mathrm{eq}}(k)\end{array}\right]^{T} \triangleq\left[\begin{array}{lll}y(k) & y(k-1)\end{array}\right.$ $\left.u_{\text {net }}(k-1)\right]^{T}$, we have

$$
\begin{aligned}
x_{1}^{\mathrm{eq}}(k+1) & =\hat{f}_{\text {narx }}\left(x_{1}^{\mathrm{eq}}(k), x_{2}^{\mathrm{eq}}(k), x_{3}^{\mathrm{eq}}(k), u_{\text {net }}(k)\right) \\
x_{2}^{\mathrm{eq}}(k+1) & =x_{1}^{\mathrm{eq}}(k) \\
x_{3}^{\mathrm{eq}}(k+1) & =u_{\text {net }}(k) \\
y(k) & =\left[\begin{array}{lll}
1 & 0 & 0
\end{array}\right] x^{\mathrm{eq}}(k)=C_{\text {narx }}\left(x^{\mathrm{eq}}(k)\right) .
\end{aligned}
$$

The system of equations in (9) can be compactly expressed in SS form as

$$
\begin{aligned}
x^{\mathrm{eq}}(k+1) & =\tilde{f}_{\text {narx }}\left(x^{\mathrm{eq}}(k), u_{\text {net }}(k)\right) \\
y(k) & =C_{\text {narx }}\left(x^{\mathrm{eq}}(k)\right)
\end{aligned}
$$

where

$$
\begin{aligned}
& \tilde{f}_{\text {narx }}\left(x^{\mathrm{eq}}(k), u_{\text {net }}(k)\right) \\
& =\left[\begin{array}{c}
\hat{f}_{\text {narx }}\left(x_{1}^{\mathrm{eq}}(k), x_{2}^{\mathrm{eq}}(k), x_{3}^{\mathrm{eq}}(k), u_{\text {net }}(k)\right) \\
x_{1}^{\mathrm{eq}}(k) \\
u_{\text {net }}(k)
\end{array}\right] .
\end{aligned}
$$

Neural network models might have inferior extrapolation behavior when operating in untrained region, and hence it is very important to validate the model with extra validation data sets, in addition to the used identification data. The identification-validation input data sets used and the corresponding outputs generated by the emulator model are shown in Fig. 3. The data cover one year with a sampling time of $4 \mathrm{~h}$. The identification input $\left(u_{\mathrm{id}}\right)$ consists of 30 multisines with frequencies distributed in the range $\left[0,0.5 f_{s}\right]$, where $f_{s}$ is the 
sampling frequency. The identification output is $y_{\mathrm{id}}=T_{f}$. The prediction performance of NARX versus the emulator model both for identification and validation data sets (where the validation input is again a multisine) for different time intervals is given in Fig. 4. The prediction performance can be expressed in terms of normalized root-mean-square error (NRMSE)

$$
\text { fit }_{\text {NRMSE }}=100 \times\left(1-\frac{\|y-\hat{y}\|}{\|y-\operatorname{mean}(y)\|}\right) .
$$

The one-step prediction performances of the NARX model corresponding to the identification and validation data sets are $\mathrm{fit}_{\mathrm{NRMSE}}^{\mathrm{id}}=98.72 \%$ and $\mathrm{fit}_{\mathrm{NRMSE}}^{\mathrm{val}}=98.63 \%$, respectively, and they are almost perfect. The simulation performance of NARX is also, compared with the emulator model, presented in Fig. 5. For the use of NARX in DP-based control, however, one-step prediction performance of NARX is relevant.

\section{B. State-Space ROM}

The emulator model given by (6) is not appropriate for an SS-based control design like MPC due to two reasons: 1 ) it is a large-scale model (506 states) and 2) its sampling time is very short: $1 \mathrm{~s}$. As a next step, a reduced-order model from the large-scale model given by (6) is obtained using the POD model order reduction technique [23], [24]. The reducedorder model is given by

$$
\begin{aligned}
x_{r}(k+1) & =A_{r} x_{r}(k)+B_{r} u_{\text {net }}(k) \\
y(k) & =T_{f}(k)=C_{r} x_{r}(k)
\end{aligned}
$$

and the details can be found in [11].

POD is a flexible model order reduction method compared with other model order reduction methods. It is based on extracting dominant system features and then projecting the large-scale dynamics on the space spanned by the dominant features. The dominant features are eigenvectors of the snapshot matrix, a matrix whose columns are state values at different time points. One of the main features of POD is that it can be applied to both linear and nonlinear ODEs. Another flexibility in POD is the ability to reflect both short- and longterm effects in the reduced-order model by the adjustment of the time instants in the construction of the snapshot matrix. The only constraint for a successful POD-based reducedorder modeling is that the large-scale model of the underlying system should be accurate. There is no limit on the level of detail. The large-scale model can even be a nonlinear model such as the Navier-Stokes equation.

Although the model in (11) is a reduced-order model (thus, smaller scale), the second issue is not yet solved: the sampling period of (11) is the same as that of the large-scale model: $1 \mathrm{~s}$. The next objective is to obtain an SS model from (11) with a larger sampling period: $4 \mathrm{~h}$. To make the discussion general, assume that (11) has a sampling period $t_{s}$ and we apply to this system an input that changes its value just over a larger sampling period $t_{L}$ and assume that $\left(t_{L} / t_{S}\right)=M>1$, where $M$ is an integer. After applying some simple algebra, one can show that the SS model with sampling period $t_{L}$

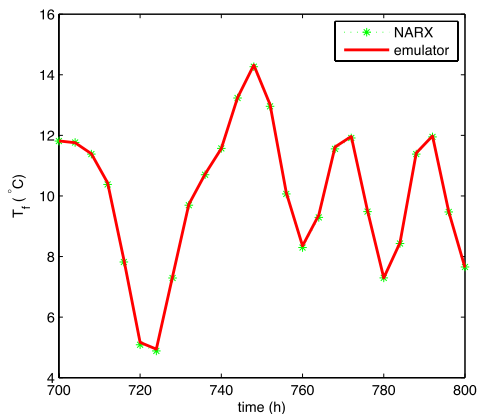

(a)

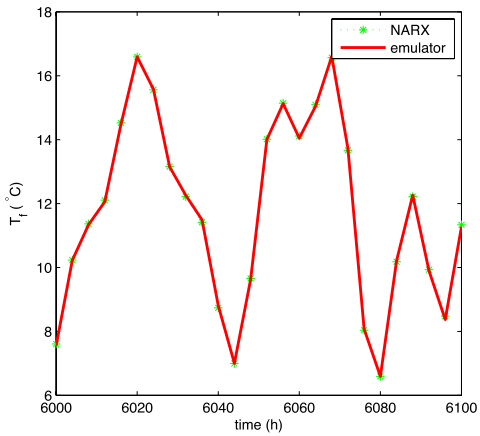

(b)

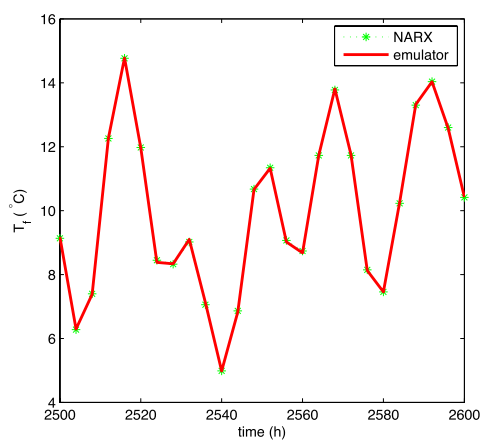

(c)

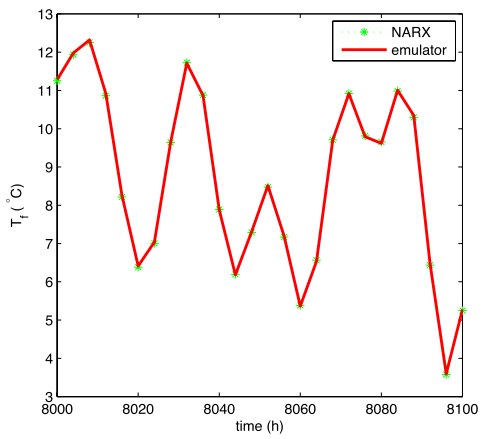

(d)

Fig. 4. Prediction performance (one step) of NARX model versus emulator model. (a) and (b) Identification input of duration: $(700-800 \mathrm{~h})$ and (6000-6100 h). (c) and (d) Validation input of duration: (2500-2600 h) and $(8000-8100 \mathrm{~h})$

(larger sampling period) is

$$
\begin{aligned}
x(k+1) & =\bar{A} x(k)+\bar{B} u_{\text {net }}(k) \\
y(k) & =\bar{C} x(k)
\end{aligned}
$$




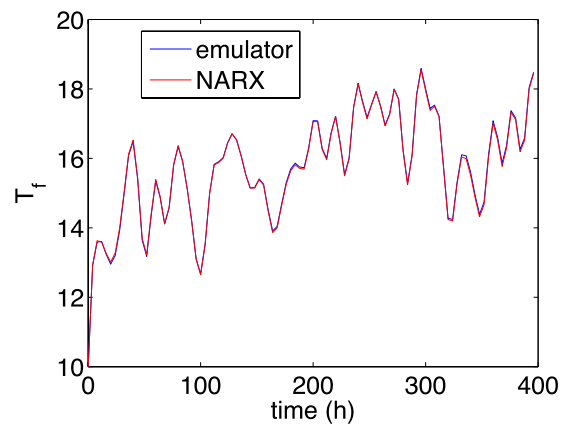

Fig. 5. Simulation performance of NARX with respect to the emulator model.

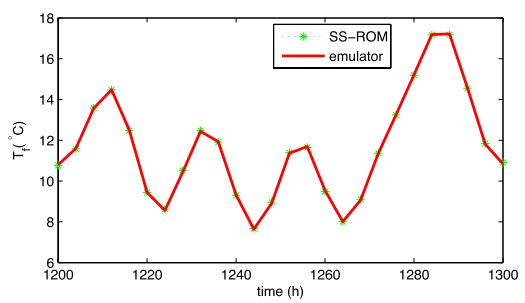

(a)

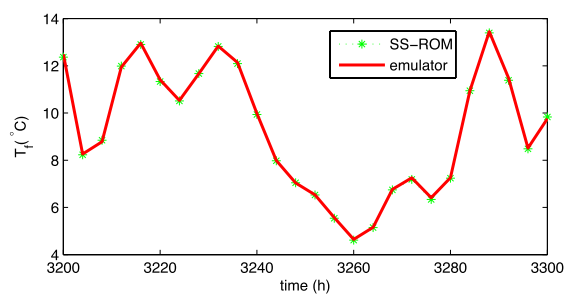

(b)

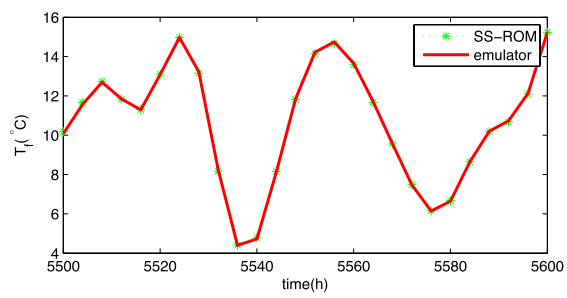

(c)

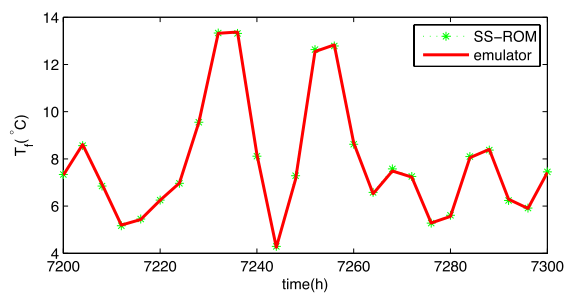

(d)

Fig. 6. Prediction performance (six steps ahead) of SS-ROM model versus emulator model. (a) and (b) Identification input of duration (1200-1300 h) and (3200-3300 h). (c) and (d) Validation input of duration (5500-5600 h) and $(7200-7300 \mathrm{~h})$.

where $\bar{A}=A_{r}^{M}, \bar{B}=\left(A_{r}^{M-1}+A_{r}^{M-2}+\cdots+I\right) B_{r}$, and $\bar{C}=C_{r}$. Now, the model given in (12) is both an SS-ROM and a model with the desired larger sampling period $t_{L}$. We denote this SS model obtained from the emulator model by SS-ROM. This model will be used in Section V-B for NMPC of the emulator system.

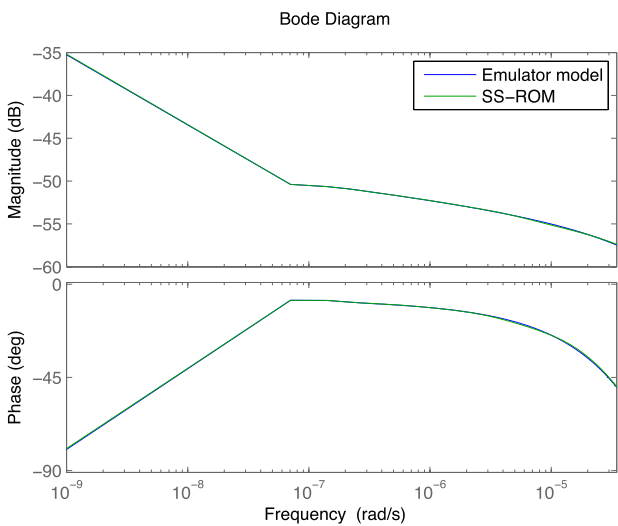

Fig. 7. Frequency response comparison of borehole emulator model and SS-ROM.

We use the same identification-validation data as in Section IV-A. The model order for SS-ROM was chosen to be six, the minimum order for accurate prediction. The prediction performance of SS-ROM versus the emulator model both for identification data set (used in POD phase) and validation data set for different time intervals of $100 \mathrm{~h}$ is shown in Fig. 6. The six-step ahead prediction performances of the SS-ROM for identification and validation data are $\mathrm{fit}_{\mathrm{NRMSE}}^{\mathrm{id}}=98.54 \%$ and $\mathrm{fit}_{\mathrm{NRMSE}}^{\mathrm{val}}=98.55 \%$, respectively. The prediction performance of the SS-ROM is almost perfect, as was also the case for the NARX model. The comparison of the frequency responses of the emulator model and the SS-ROM is shown in Fig. 7. Note that the Nyquist frequency of the discrete-time emulator and SS-ROM models is $0.5 \times 1 /(4 \times 3600)=3.4722 \times 10^{-5} \mathrm{~Hz}$.

\section{Optimal Control Approaches AND COMPARISON OF RESULTS}

In this section, DP, NMPC, and LOC formulations of the HyGCHP system will be presented as general as possible, but the results without taking into account zero TBUD constraint and assuming zero building load violation margins will be shown. The reason for such a restriction was to be able to apply DP, MPC, and LOC under the same set of constraints and, hence, to compare the results fairly. The control results under the constraint of prevention of TBUD in the ground and inclusion of load violation margins will be presented in the next sections for MPC and LOC approaches. In all the simulations of the controlled system, controllers were applied on the detailed (finite-volume) emulator model. The platforms for identification, optimization, and simulation were MATLAB system identification toolbox, MATLAB optimization toolbox, and MATLAB Simulink, respectively.

\section{A. Dynamic Programming-Based Optimal Control}

Consider the expression inside the summation given in cost function (1)

$$
\begin{aligned}
h=t_{s}[ & c_{e}(k)\left(\frac{\dot{Q}_{\mathrm{hp}}(k)}{\operatorname{COP}_{\mathrm{hp}}(k)}+\frac{\dot{Q}_{\mathrm{pc}}(k)}{\operatorname{COP}_{\mathrm{pc}}(k)}+\frac{\dot{Q}_{\mathrm{ch}}(k)}{\operatorname{COP}_{\mathrm{ch}}(k)}\right) \\
& \left.+c_{g} \frac{\dot{Q}_{\mathrm{gb}}(k)}{\eta_{\mathrm{gb}}}\right] .
\end{aligned}
$$


The electricity price $c_{e}(k)$ varies according to $\mathrm{D} / \mathrm{N}$ tariff with 0.09 euro/kWh for 8 A.M-10 P.M., 0.15 euro/kWh for rest, and $c_{g}$ is taken 0.06 euro/kWh. Here, COPs and efficiencies are fitted to TRNSYS data [25]

$$
\begin{aligned}
\operatorname{COP}_{\mathrm{hp}}(k) & =\alpha_{0}+\alpha_{1} T_{f}(k)+\alpha_{2} T_{f}^{2}(k) \\
\operatorname{COP}_{\mathrm{ch}}(k) & =\beta_{0}+\beta_{1} T_{a}(k)+\beta_{2} T_{a}^{2}(k) \\
\mathrm{COP}_{\mathrm{pc}} & =12, \quad \eta_{\mathrm{gb}}=1
\end{aligned}
$$

where $\mathrm{COP}_{h p}$ and $\mathrm{COP}_{c h}$ are fitted for a supply water temperature of $T_{\mathrm{sw}, h}=40{ }^{\circ} \mathrm{C}$ for heating and a supply water temperature of $T_{\mathrm{sw}, c}=18{ }^{\circ} \mathrm{C}$ for cooling, typically coupled to thermally activated building systems. The coefficients are $\alpha_{0}=3.04, \alpha_{1}=0.079, \alpha_{2}=-0.0012 ; \beta_{0}=10.22$, $\beta_{1}=-0.26$, and $\beta_{2}=0.002$. Using (10b), (14a) can be written as

$$
\begin{aligned}
\operatorname{COP}_{\mathrm{hp}}(k) & =\alpha_{0}+\alpha_{1} C_{\mathrm{narx}}\left(x^{\mathrm{eq}}(k)\right)+\alpha_{2} C_{\mathrm{narx}}^{2}\left(x^{\mathrm{eq}}(k)\right) \\
& \triangleq f_{\mathrm{COP}_{\mathrm{hp}}}\left(x^{\mathrm{eq}}(k)\right) .
\end{aligned}
$$

The requirement of exact heating and cooling load satisfaction gives

$$
\begin{aligned}
& \dot{Q}_{\mathrm{gb}}(k)=\dot{Q}_{h}(k)-\dot{Q}_{\mathrm{hp}}(k) \\
& \dot{Q}_{\mathrm{pc}}(k)=\dot{Q}_{c}(k)-\dot{Q}_{\mathrm{ch}}(k) .
\end{aligned}
$$

Using (16), (13) becomes

$$
\begin{aligned}
h= & t_{s}\left[\frac{c_{e}(k)}{\operatorname{COP}_{\mathrm{hp}}(k)}-\frac{c_{g}(k)}{\eta_{\mathrm{gb}}}\right] \dot{Q}_{\mathrm{hp}}(k) \\
& +t_{s}\left[\frac{c_{e}(k)}{\operatorname{COP}_{\mathrm{ch}}(k)}-\frac{c_{e}(k)}{\mathrm{COP}_{\mathrm{pc}}}\right] \dot{Q}_{\mathrm{ch}}(k) \\
& +t_{s}\left[\frac{c_{g}(k)}{\eta_{\mathrm{gb}}} \dot{Q}_{h}+\frac{c_{e}(k)}{\mathrm{COP}_{\mathrm{pc}}} \dot{Q}_{c}\right] .
\end{aligned}
$$

We assumed satisfaction of exact heating-cooling demands (zero violation margins) to formulate the DP with two control inputs $\left(\dot{Q}_{\mathrm{hp}}\right.$ and $\left.\dot{Q}_{\mathrm{ch}}\right)$ instead of four $\left(\dot{Q}_{\mathrm{hp}}, \dot{Q}_{\mathrm{ch}}, \dot{Q}_{\mathrm{gb}}\right.$, and $\dot{Q}_{\mathrm{pc}}$ ) to alleviate a numerical difficulty of DP (known as curse of dimensionality; more details will be given in the following).

Note that we can express $h$ in (17) as $h=h(y, u, w)$, where $u=\left[\dot{Q}_{\mathrm{hp}} \dot{Q}_{\mathrm{ch}}\right]^{T}, y=T_{f}$ is the circulating fluid mean temperature, and $w=\left[\begin{array}{lllll}c_{e} & c_{g} & T_{a} & Q_{h} & Q_{c}\end{array}\right]^{T}$.

Next, we will express the borehole dynamics (10) in terms of the $u$ and $w$. Using (4) and (16), the net input to the ground can be expressed as

$$
\begin{aligned}
u_{\mathrm{net}} & =\frac{\mathrm{COP}_{\mathrm{pc}}+1}{\mathrm{COP}_{\mathrm{pc}}}\left[\dot{Q}_{c}-\dot{Q}_{\mathrm{ch}}\right]-\frac{\mathrm{COP}_{\mathrm{hp}}-1}{\mathrm{COP}_{\mathrm{hp}}} \dot{Q}_{\mathrm{hp}} \\
& =\frac{\mathrm{COP}_{\mathrm{pc}}+1}{\mathrm{COP}_{\mathrm{pc}}}\left[\dot{Q}_{c}-\dot{Q}_{\mathrm{ch}}\right]-\frac{f_{\mathrm{COP}_{\mathrm{hp}}}\left(x^{\mathrm{eq}}(k)\right)-1}{f_{\mathrm{COP}_{\mathrm{hp}}}\left(x^{\mathrm{eq}}(k)\right)} \dot{Q}_{\mathrm{hp}} \\
& \triangleq f_{u_{\mathrm{net}}}\left(x^{\mathrm{eq}}, u, w\right) .
\end{aligned}
$$

Then, the borehole dynamics (10) can be expressed as

$$
\begin{aligned}
x^{\mathrm{eq}}(k+1) & =\tilde{f}_{\text {narx }}\left(x^{\mathrm{eq}}(k), u_{\text {net }}(k)\right) \\
& =\tilde{f}_{\text {narx }}\left(x^{\mathrm{eq}}(k), f_{u_{\text {net }}}\left(x^{\mathrm{eq}}(k), u(k), w(k)\right)\right) \\
& \triangleq f_{\text {narx }}\left(x^{\mathrm{eq}}(k), u(k), w(k)\right) \\
y(k) & =C_{\text {narx }}\left(x^{\mathrm{eq}}(k)\right) .
\end{aligned}
$$

Now, the DP formulation of the control problem can be given: minimization of total energy cost (1) under operational constraints. DP is based on the principle of optimality [26], which simply says that in a multistage process whatever the previous states are, the remaining decisions must be optimal with regard to the state following from the current state. This principle allows the optimal control problem for a $K$-stage process to be recursively formulated starting from the final discrete time point $N$

$$
\begin{aligned}
& J_{N-K, N}^{\star}(y(N-K)) \\
& =\min _{u(N-K)}\left\{h(y(N-K), u(N-K), w(N-K))+J_{N-(K-1), N}^{\star}\right. \\
& \times(h(y(N-(K-1)), w(N-(K-1))))\} .
\end{aligned}
$$

Using the SS dynamics given in (19), we can write (20) as

$$
\begin{aligned}
& J_{N-K, N}^{\star}\left(x^{\mathrm{eq}}(N-K)\right) \\
&=\min _{u(N-K)}\{ h\left(C_{\text {narx }}\left(x^{\mathrm{eq}}(N-K)\right), u(N-K), w(N-K)\right) \\
&+J_{N-(K-1), N}^{\star}\left(h \left(C _ { \text { narx } } \left(f _ { \text { narx } } \left(x^{\mathrm{eq}}(N-K),\right.\right.\right.\right. \\
&u(N-K)), w(N-K)), w(N-K+1))\} .
\end{aligned}
$$

Here, $J_{N-K, N}^{\star}$ is the optimal cost for a $K$-stage policy with the initial state $x^{\mathrm{eq}}(N-K)$ and $J_{N-(K-1), N}^{\star}$ is the optimal cost for a $K-1$-stage policy. To start the algorithm, we set $J_{N, N}^{\star}=0$, which is the cost of stage number zero, $K=0$.

Typically, in DP, the feasible states and feasible inputs are gridded into quantized values and then at a given stage with a chosen feasible state value, and all possible quantized inputs are tried until the minimum of (21) is determined at that stage. This is done for all feasible gridded states. Hence, the DP control algorithm is a closed-loop and global optimal control algorithm (global optimal up to the approximations due to state-input gridding and interpolations). In the NARX model (8), the variables in the role of states are $\left\{y(k), y(k-1), u_{\text {net }}(k-1)\right\}$ and the input to be determined is $u_{\text {net }}(k)$ at time step $k$. Note that in DP-based control, instead of SS-ROM (with six states), we preferred an input-output model NARX (with equivalent three states) due to the curseof-dimensionality problem [26], [27].

In the optimal control problem, the control input range for both $\dot{Q}_{\mathrm{hp}}$ and $\dot{Q}_{\mathrm{ch}}$ is taken to be $[0,6000] \mathrm{W}$ and is gridded into 300 points. The range for output $y$ is taken as $[0.5,19.5]^{\circ} \mathrm{C}$, and it is gridded into 200 points. The results are shown for all relevant variables for a heating-dominated case in Fig. 8, for a cooling-dominated case in Fig. 9, and finally, for a thermally balanced case in Fig. 10, where

$$
r_{1}(k) \triangleq \frac{c_{e}(k) / \mathrm{COP}_{\mathrm{hp}}(k)}{c_{g}(k) / \eta_{\mathrm{gb}}}, \quad r_{2}(k) \triangleq \frac{c_{e}(k) / \mathrm{COP}_{\mathrm{ch}}(k)}{c_{e}(k) / \mathrm{COP}_{\mathrm{pc}}} .
$$

Observe from (17) that $r_{1}$ determines whether instantaneously it is advantageous to use the hp or gb and $r_{2}$ whether to use the ch or pc. $r_{1}<1$ means to choose the hp over gb and $r_{2}<1$ to choose the ch over pc (under the assumption that power limits and $T_{f}$ bounds are not violated). From Figs. 8(d) and (e)-10(d) and (e), we observe that always 


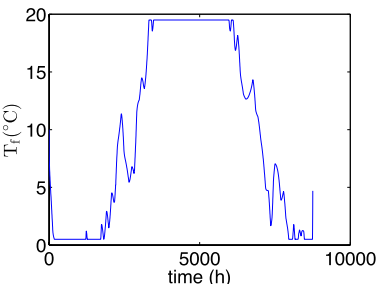

(a)

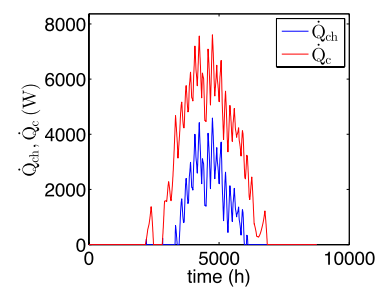

(c)

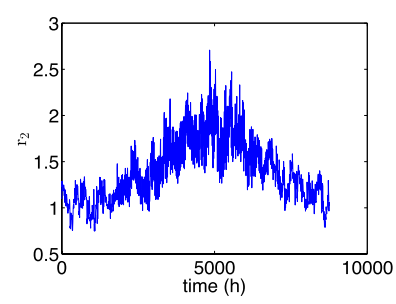

(e)

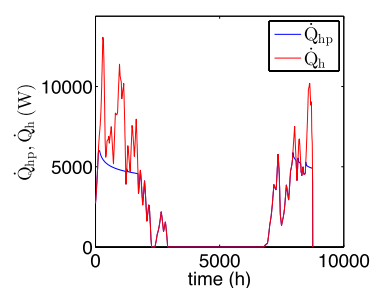

(b)

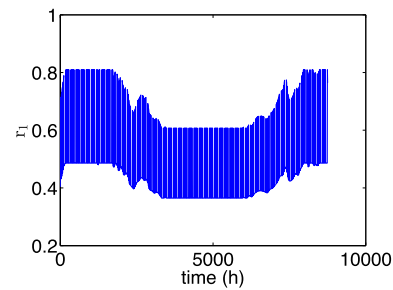

(d)

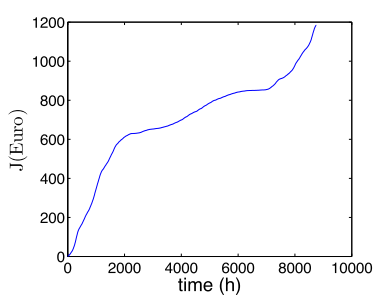

(f)

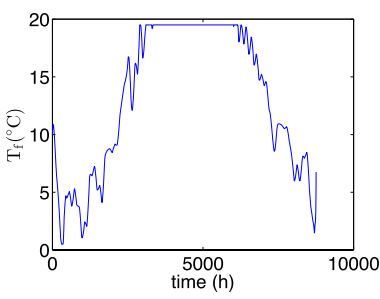

(a)

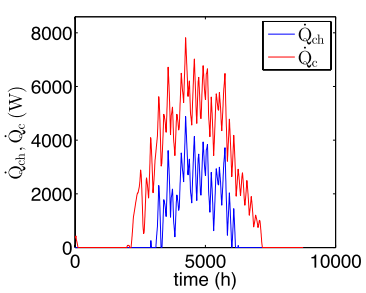

(c)

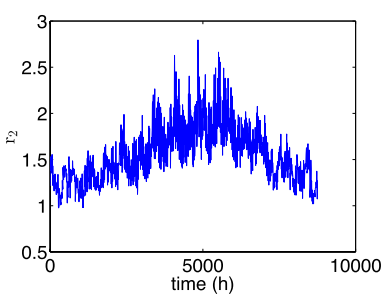

(e)

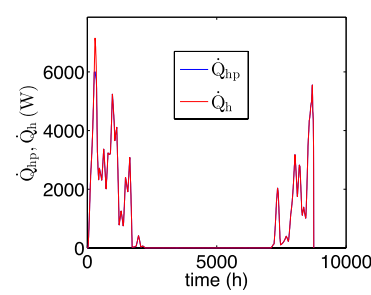

(b)

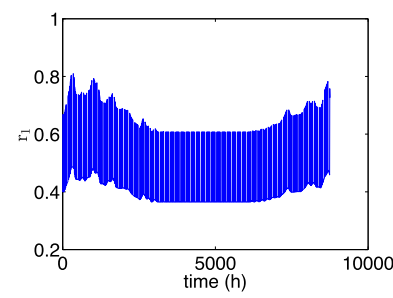

(d)

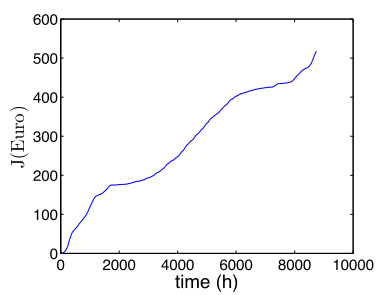

(f)

Fig. 8. Controlled system variables for heating-dominated case (DP). (a) Circulating fluid mean temperature. (b) Heat demand and hp power. (c) Cold demand and ch power. (d) $r_{1}$. (e) $r_{2}$. (f) Accumulated cost profile.

$r_{1}<1$ but $r_{2}>1$ most of the time. Indeed, in the heatingdominated and balanced cases, $r_{2}$ is always greater than one when cooling is required because when $r_{2}<1$, there is no cooling load. For the cooling-dominated case, $r_{2}>1$. As a result, for all three categories, we can say that it is always advantageous to use passive cooling, which is expected since the pc has a higher efficiency than the ch. Note that the $\dot{Q}_{\mathrm{hp}}$ value is equal to the demanded heat value as long as the hp capacity is not exceeded or the circulating fluid mean temperature is not crossing the temperature bounds. When the temperature bounds are approached or reached, a gradual decrease in $\dot{Q}_{\mathrm{hp}}$ is observed and the remaining heating load should be provided by the gb. A similar behavior holds for $\dot{Q}_{\text {ch }}$ : as long as the circulating fluid mean temperature bounds are not approached, the pc is used. As a result, the critical control actions are totally determined by: 1) power capacity of the hp and ch and 2) temperature bounds for $T_{f}$. This observation may suggest that these important parameters may be used in a rule-based control strategy without needing a model-based intelligent control algorithm. This is not the case because, for example, when $T_{f}$ temperature bounds are approached, it is very difficult to decide the power ratio between $\mathrm{hp} / \mathrm{gb}$ in the heating case and the power ratio between $\mathrm{ch} / \mathrm{pc}$ in the cooling case in order not to exceed but stay close to these temperature bounds. In Figs. 8(b) and (c)10(b) and (c), the difference between required loads (red color) and the part provided either by hp or ch (blue color) means

Fig. 9. Controlled system variables for cooling-dominated case (DP). (a) Circulating fluid mean temperature. (b) Heat demand and hp power. (c) Cold demand and ch power. (d) $r_{1}$. (e) $r_{2}$. (f) Accumulated cost profile.

that these differences should be provided by the gb for heating cases and by the pc for cooling cases.

\section{B. Nonlinear Model Predictive Control of the System}

First, the main aspects of NMPC are presented. Given general process dynamics

$$
x(k+1)=f(x(k), u(k), w(k))
$$

subject to the general constraint function including bounds on input and state variables

$$
g(x(k), u(k), w(k)) \leq 0
$$

the objective is the minimization of

$$
J(x(k), \bar{u}(k), w(k))=\sum_{i=k}^{k+N_{p}} h(\bar{x}(i), \bar{u}(i), w(i))
$$

where

$$
\bar{x}(k+1)=f(\bar{x}(k), \bar{u}(k), w(k)), \quad \bar{x}(k)=x(k)
$$

under the constraints

$$
\begin{array}{rlrl}
g(\bar{x}(k), \bar{u}(k), w(k)) & \leq 0 \\
\bar{u}(s) & =\bar{u}\left(k+N_{c}\right), & k+N_{c} \leq s \leq k+N_{p} \\
& N_{c} \leq N_{p} .
\end{array}
$$




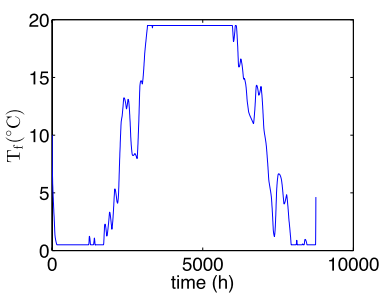

(a)

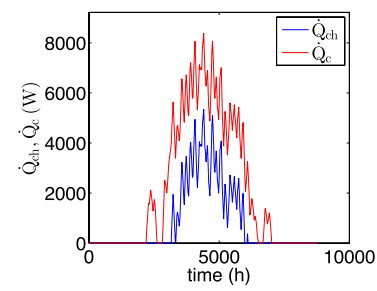

(c)

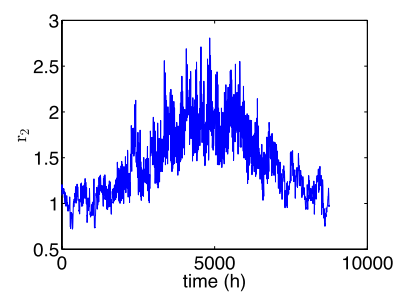

(e)

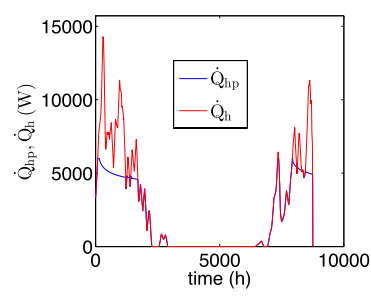

(b)

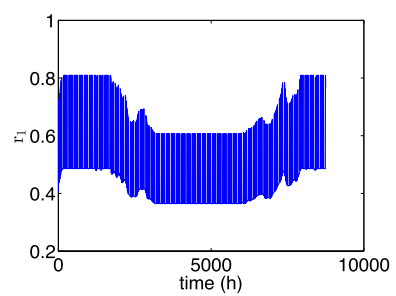

(d)

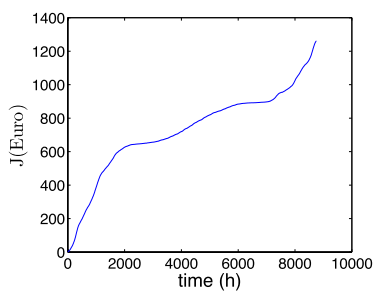

(f)
Fig. 10. Controlled system variables for thermally balanced case (DP). (a) Circulating fluid mean temperature. (b) Heat demand and hp power. (c) Cold demand and ch power. (d) $r_{1}$. (e) $r_{2}$. (f) Accumulated cost profile.

Here, $N_{p}$ and $N_{c}$ are called prediction and control horizons, respectively. The bar notation denotes the internal control variables (the calculated control inputs) and the resulting predicted states over the given horizons. A distinction between the real system variables and the variables in the controller (denoted with a bar) is necessary because the calculated inputs and the predicted values may not be the same as the ones in the real-time controlled system. Moreover, NMPC is applied in a receding horizon way, meaning that only the first input of the calculated input sequence is applied and the optimization is redone at every time step.

For the NMPC, we will use the SS model SS-ROM constructed in Section IV-B. In the HyGCHP system model, the nonlinearity enters in the objective function and in the input part of the system dynamics $\left[\dot{Q}_{\text {net }}=\dot{Q}_{\text {inj }}-\dot{Q}_{\text {ext }}\right.$, see (4)] due to the state dependency of hp COP: $\operatorname{COP}_{\mathrm{hp}}\left(T_{f}\right)$ [see (14)]. We choose $N_{p}=N_{c}=6$ (24-h prediction and control horizon), and in minimization of (24), a multisearch global optimization scheme is used to force the solution toward a global optimal solution. Seeking for a global optimal solution is important since we will compare the results with the DP results from Section V-A. In the implementation of the NMPC controller, an observer is designed for the estimation of SS-ROM states from the input-output of the emulator model. The results are almost indistinguishable from the results of DP (Figs. 8-10), and hence the very similar plots will not be repeated here. This is a very interesting observation, and it means that MPC-based energy minimization of the system is almost the same as its DP-based minimization over a period of time (here one year). In other words, the total energy minimization problem does not depend on future inputs, states, and disturbances. This observation is very important because in most applications of GCHPs with supplementary devices, the control problem is defined as keeping the building zone temperature(s) in a thermal comfort band and this control policy is applied online. Now, after the above observation, this control problem can be solved in an energy-optimal way: online minimize the total energy use of the system over the MPC horizon and put temperature bounds for thermal comfort as constraints. This policy will determine a control trajectory that ensures thermal comfort while the total use of energy will be minimized over the system operation period, one year in our case. This observation for the studied energy-optimal control of a HyGCHP system, in fact, raises the following general theoretical optimal control research problem-assume that we have an optimal control problem:

$$
\begin{array}{ll}
\min & \sum_{k=1}^{N} h(x(k), u(k), w(k)) \\
\text { s.t. } & x(k+1)=A x(k)+B(x(k)) u(k) \text { and } \\
& g(x(k), u(k), w(k)) \leq 0 .
\end{array}
$$

Then, what are the necessary and/or sufficient conditions so that the optimal solution will not depend on future control/ states/disturbances? An answer to this general problem is very important for the studied control problem in this paper to understand why total energy minimization over the whole control period was very weakly dependent on future variables. However, this theoretical problem is out of the scope of this paper and it is the topic of a future research. Note that the objective of this section was to compare performance of NMPC with DP. Since NMPC cannot see the whole control period (which is one year), zero TBUD in the ground was not considered as a constraint in DP-based control. It will be taken into account as a constraint in the next sections.

\section{Linear Optimal Control of the System}

In this section, we take the COP value of the hp constant, and hence make the objective function and equivalent borehole dynamics of the control problem linear. The COP of the ch is variable as was assumed before and has no impact on linearity of the system (it is not state or control input dependent). We apply the classical LOC theory (minimization of energy over a given period of time) and consider the objective function with two control inputs $u=\left[Q_{\mathrm{hp}}, Q_{\mathrm{ch}}\right]^{T}$, which is obtained summing (17) over a period

$$
\begin{aligned}
J=t_{s} \sum_{k=0}^{N-1}\{ & {\left[\frac{c_{e}(k)}{\mathrm{COP}_{\mathrm{hp}}=\text { constant }}-\frac{c_{g}(k)}{\eta_{\mathrm{gb}}}\right] \dot{Q}_{\mathrm{hp}}(k) } \\
+ & {\left[\frac{c_{e}(k)}{\mathrm{COP}_{\mathrm{ch}}(k)}-\frac{c_{e}(k)}{\mathrm{COP}_{\mathrm{pc}}}\right] \dot{Q}_{\mathrm{ch}}(k) } \\
& \left.+\left[\frac{c_{g}(k)}{\eta_{\mathrm{gb}}} \dot{Q}_{h}+\frac{c_{e}(k)}{\mathrm{COP}_{\mathrm{pc}}} \dot{Q}_{c}\right]\right\}
\end{aligned}
$$




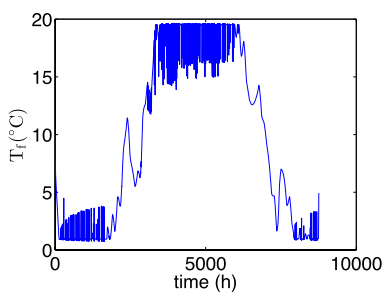

(a)

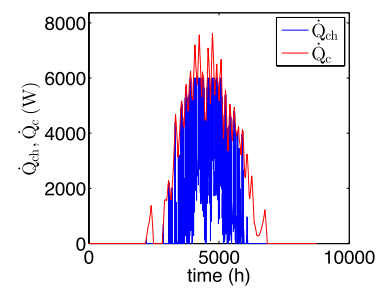

(c)

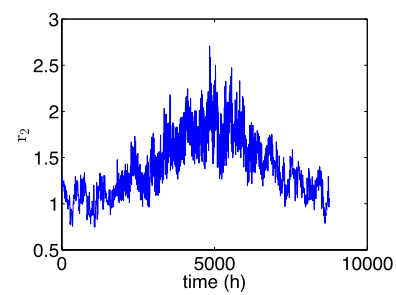

(e)

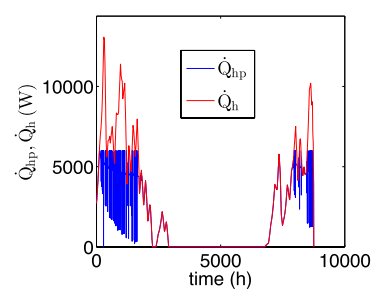

(b)

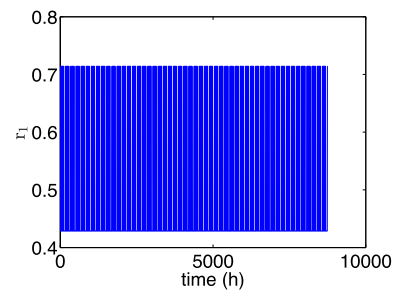

(d)

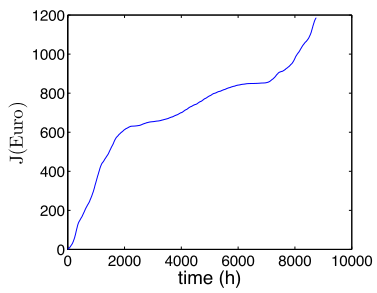

(f)

Fig. 11. Controlled system variables for heating-dominated case (LOC with $\mathrm{COP}_{\mathrm{hp}}=3.5$ ). (a) Circulating fluid mean temperature. (b) Heat demand and hp power. (c) Cold demand and ch power. (d) $r_{1}$. (e) $r_{2}$. (f) Accumulated cost profile.

The function in (27) will be minimized over a one-year period using the operational constraints given by (2)-(5) and the SS-ROM model dynamics. The typical range of $\mathrm{COP}_{\mathrm{hp}}$ is taken as $[2.5,6]$. This range is gridded into a set of grid points with a grid size of 0.1 . Then, for each grid point, a corresponding $\mathrm{LOC}$ is designed. The results for $\mathrm{COP}_{\mathrm{hp}}=3.5$ are shown in Figs. 11-13.

From Figs. 11-13, we observe that the results are close to the DP and NMPC cases with some differences: 1) more oscillation in hp and ch power and this in turn causes and 2) the circulating fluid mean temperature to become oscillatory, especially when the temperature bounds are approached.

The annual cost, maximum lower, and upper temperature violation of $T_{f}$ versus $\mathrm{COP}_{\mathrm{hp}}$ are shown in Fig. 14. The total cost is compared with the total cost in the DP $(\approx$ NMPC) case. A zero temperature bound violation for either lower bound or upper bound of $T_{f}$ means that the corresponding bound is not exceeded. The results show that taking any reasonable constant COP for hp gives more or less a similar annual cost, but $T_{f}$ temperature bounds are exceeded. A $\mathrm{COP}_{\mathrm{hp}}$ value in the range [3,4] gives the results for which $T_{f}$ temperature bound violation is acceptable. Note that for some $\mathrm{COP}_{h p}$ values, the annual costs of LOC cases are slightly lower than the corresponding cases of DP. This may seem strange since the DP case was a global optimal approach. This can be explained by a violation of the temperature bounds in the LOC cases.

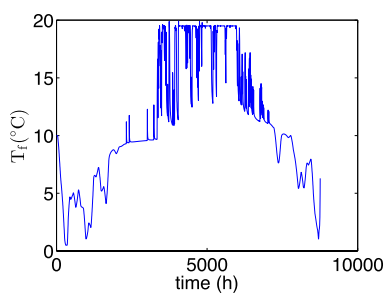

(a)

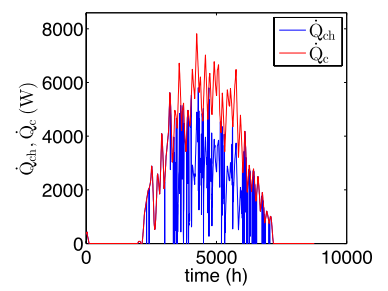

(c)

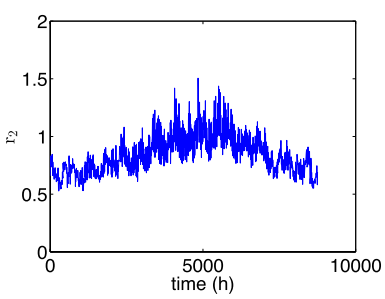

(e)

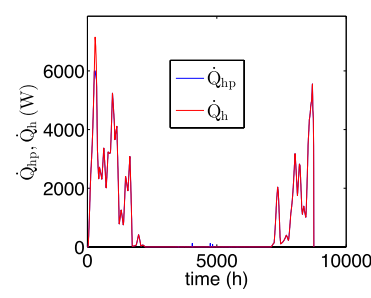

(b)

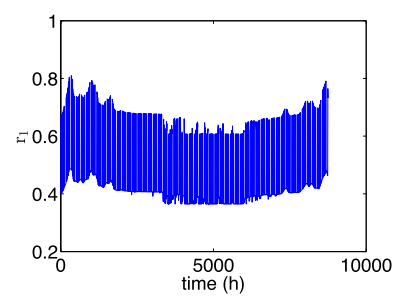

(d)

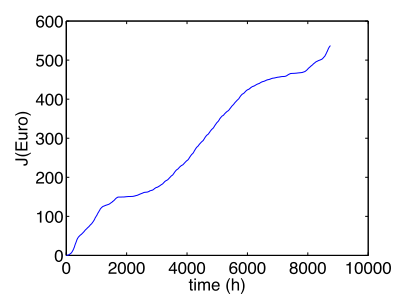

(f)
Fig. 12. Controlled system variables for cooling-dominated case (LOC with $\mathrm{COP}_{\mathrm{hp}}=3.5$ ). (a) Circulating fluid mean temperature. (b) Heat demand and hp power. (c) Cold demand and ch power. (d) $r_{1}$. (e) $r_{2}$. (f) Accumulated cost profile.

TABLE I

COMPUTATION TIMES FOR CONTROLLERS FOR THE HEATING-DOMINATED CASE

\begin{tabular}{|l|l|l|}
\hline DP & NMPC & LOC \\
\hline 68 hours & 49 hours & 2 minutes \\
\hline
\end{tabular}

In fact, comparison of annual costs with the corresponding results of DP may be unreasonable under the violation of $T_{f}$ bounds. Such a comparison was done only to give an idea, and it was not meant as a real comparison.

Comparisons of computation times for DP, NMPC, and LOC controllers are presented in Table I for the heatingdominated case.

\section{DP AND LOC With Zero ThermaL BUILDUP/DEPLETION}

In Section V, we neglected TBUD in the ground during control for a fair comparison with MPC. However, this effect is important and should be taken into consideration if the system has to operate for a long term. From the results of Section V, we calculate a thermal depletion of approximately 5\% for the heating-dominated case (calculated as the ratio of annual net heat to the ground to annual heating load) and a thermal buildup of $40 \%$ for the cooling-dominated case (calculated as 


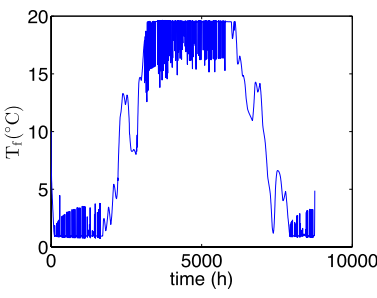

(a)

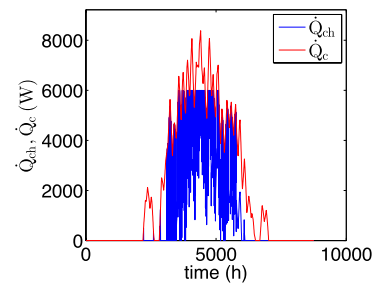

(c)

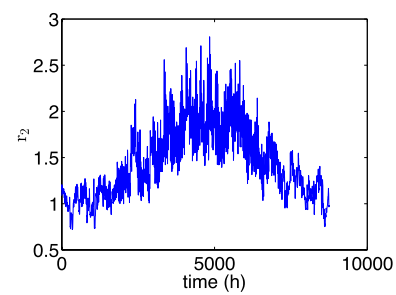

(e)

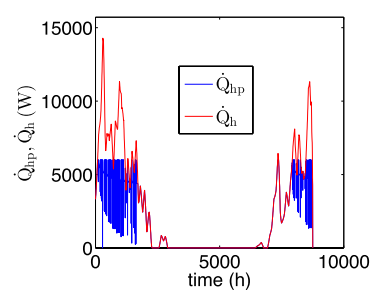

(b)

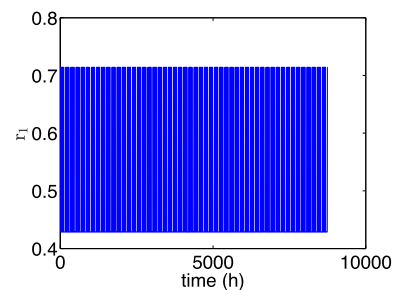

(d)

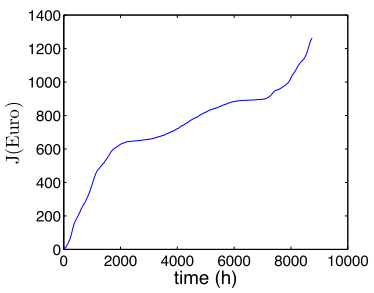

(f)
Fig. 13. Controlled system variables for thermally balanced case (LOC with $\mathrm{COP}_{\mathrm{hp}}=3.5$ ). (a) Circulating fluid mean temperature. (b) Heat demand and hp power. (c) Cold demand and ch power. (d) $r_{1}$. (e) $r_{2}$. (f) Accumulated cost profile.

the ratio of annual net heat to the ground to annual cooling load). For the thermally balanced case, TBUD was almost negligible. All the control approaches gave the results within the above bands.

In this section, we force the system to have zero TBUD in the ground. The comparison of the control results for DP and LOC for all the loading types is shown in Fig. 15, where only circulating fluid mean temperatures and accumulated cost profiles are shown to save space. The LOC results are shown for $\mathrm{COP}_{h p}=3,4$, and 5. Note that zero TBUD in the ground cannot be put as a constraint in NMPC since NMPC cannot see the whole horizon, and hence it is not relevant for this section.

The results of Fig. 15 show that circulating fluid mean temperatures become more oscillatory toward the lower-upper bounds to satisfy the zero TBUD in the ground at the end of the year. However, the trend of accumulated cost profiles is not affected considerably, when compared with the results in Section V.

\section{Optimal Control With Additional Scenarios}

In this section, we consider additional scenarios using MPC and LOC. In these scenarios, we include a new constraint for reducing accumulation of heat/cold demand dissatisfaction at
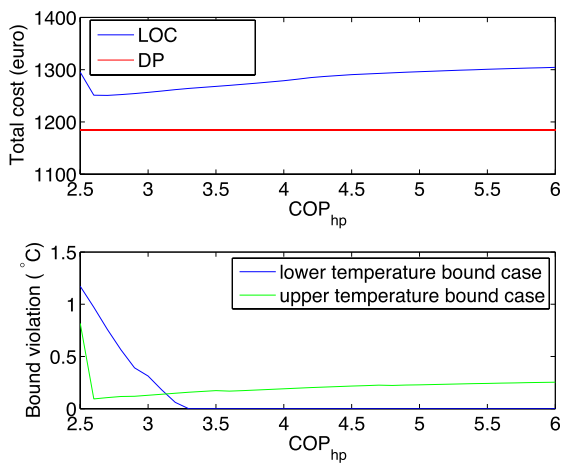

(a)
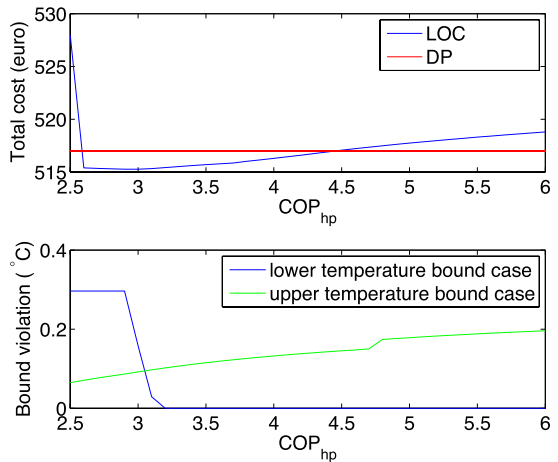

(b)
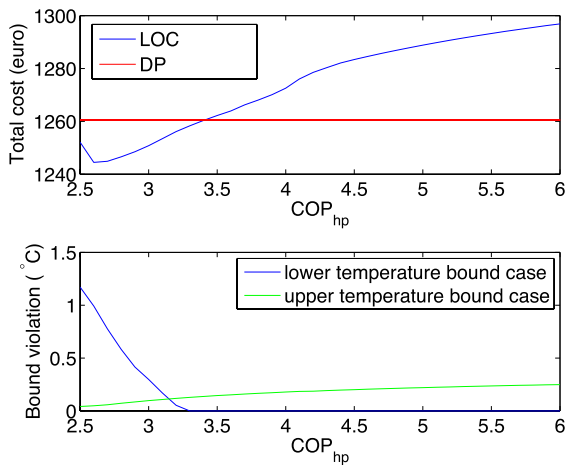

(c)

Fig. 14. Annual cost and temperature bound violation ranges (for LOC) versus $\mathrm{COP}_{\mathrm{hp}}$. (a) Heating-dominated case. (b) Cooling-dominated case. (c) Thermally balanced case.

the end of the day

$$
\begin{aligned}
& \left|\sum_{k=i}^{i+N_{p}-1} \dot{Q}_{\mathrm{hp}}(k)+\dot{Q}_{\mathrm{gb}}(k)-\dot{Q}_{h}(k)\right|=0 \\
& \left|\sum_{k=i}^{i+N_{p}-1} \dot{Q}_{\mathrm{ch}}(k)+\dot{Q}_{\mathrm{pc}}(k)-\dot{Q}_{c}(k)\right|=0
\end{aligned}
$$

where $i$ is the current time step and $N_{p}$ is the prediction horizon. This constraint will be applied both for NMPC and LOC. However, note that since NMPC is applied with receding horizon, this constraint will not guarantee the prevention of accumulated heat/cold demand dissatisfaction 


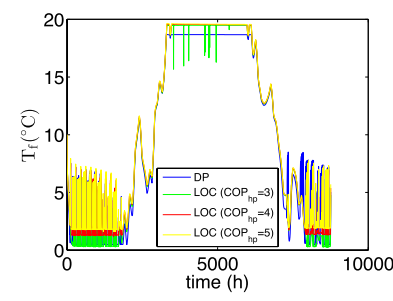

(a)

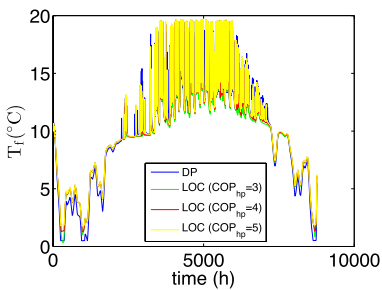

(c)

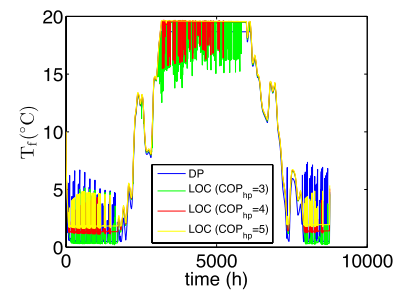

(e)

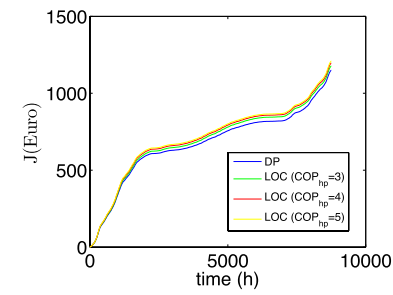

(b)

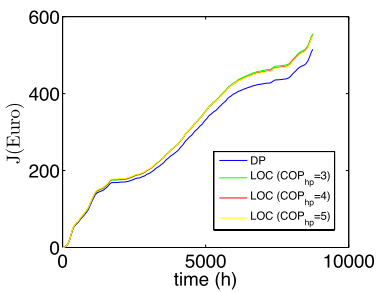

(d)

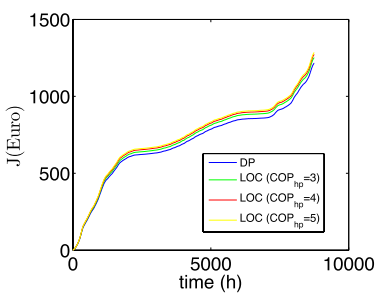

(f)
Fig. 15. Comparison of results for DP and LOC in te case of zero ground TBUD. (a) Circulating fluid mean temperature (hdom). (b) Accumulated cost profile (hdom). (c) Circulating fluid mean temperature (cdom). (d) Accumulated cost profile (cdom). (e) Circulating fluid mean temperature (bal). (f) Accumulated cost profile (bal).

at the end of the day, but it will increase the probability of reduction of accumulation.

In the previous sections, we presented the control results for MPC-LOC and compared them with DP. However, as explained before, due to the curse-of-dimensionality problem, we assumed perfect satisfaction of heat-cold demands to be able to reformulate DP control with only two control inputs, instead of four. As a result, in all the control results in the previous sections, we set violation margins to zero. In this section, we allow demand violation margins to be up to $5 \%$ of the associated heat-cold demand at a given time. Since it was not possible to control the system (due to the curseof-dimensionality issue) with DP when nonzero margins are considered, here the results are presented only for NMPC and LOC. Moreover, in this section, we consider a variable electricity price profile, in contrast to the previous $\mathrm{D} / \mathrm{N}(\mathrm{D} / \mathrm{N})$ profile. The variable electricity price profile is based on day ahead market prices from the Belgian transmission grid operator for the year 2013 [29]. However, these prices are not representative for electricity consumers, as these also have to pay a fixed cost component for transmission, distribution, taxes, and levies. In line with current electricity prices in Europe [28], this constant cost component is assumed to be $0.07 \mathrm{EUR} / \mathrm{kWh}$ and is added to the day ahead market prices. The annual variation of the variable electricity price is shown

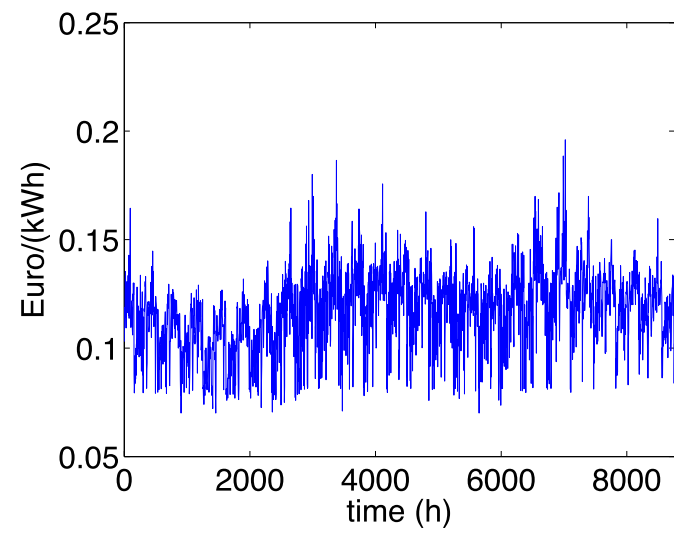

Fig. 16. Variable electricity prices [28], [29].

in Fig. 16.

Next, based on a combination of selected control method (NMPC or LOC), whether a D/N or variable electricity price profile is used, zero/nonzero demand violation margins, a set of new scenarios is created. The results of all the scenarios considered so far in the previous sections and the new ones are listed in Table II. In Table II, the annual cost results (A.cost) are compared with the base case of DP control with zero margins, D/N electricity prices, and where TBUD in the ground is allowed. This base case is denoted as a scenario with number 1 and is shown in bold face. The last column shows the RC of annual costs with respect to the base case annual cost as percentage. In the presented scenarios, all the three building types (B.type) are considered. The new scenarios are these with numbers $3-5$ and $8-10$, and in these new scenarios, the additional heat-cold accumulation constraint (28) is taken into account, in addition to variable electricity prices and/or nonzero demand violation margins. From the results in Table II, we observe the following.

1) Five percent demand violation margins with $D / N$ electricity prices reduces the annual cost up to $6.9 \%$ for NMPC and $2.2 \%$ for LOC. However, reduction in cost for LOC is dependent on the hp COP value assumed in the controller design. For some COP values, there is an increase in annual cost compared with the base case even with nonzero demand violation margins.

2) The combination of nonzero demand violation margins and variable electricity prices provided higher cost reduction in the NMPC case. The corresponding reduction results (if it happens) in the LOC case are lower and $\mathrm{COP}_{h p}$ dependent. As before, for some $\mathrm{COP}_{\mathrm{hp}}$ values, there is an increase in the annual cost relative to the base case.

3) The percentage of RC of annual cost, when 5\% violation margins and/or variable electricity prices are considered, is not more than $10 \%$ for all the cases applying NMPC or LOCs.

\section{LESSONS LEARNED FROM ANALYSIS OF OPTIMAL CONTROL APPROACHES}

From the analysis of different optimal control approaches applied to a HyGCHP system, we extract the following points. 
This article has been accepted for inclusion in a future issue of this journal. Content is final as presented, with the exception of pagination.

TABLE II

Scenarios and Comparison of Annual Costs With Base DP Control Case (Scenario \#1)

\begin{tabular}{|c|c|c|c|c|c|c|c|}
\hline Scenario \# & Controller & Margin & E. Price & TBUD & B. Type & A.Cost & $\mathbf{R C}(\%)$ \\
\hline \multirow{3}{*}{ 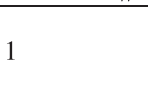 } & \multirow{3}{*}{ DP (NMPC) } & \multirow{3}{*}{ zero } & \multirow{3}{*}{$\mathbf{D} / \mathbf{N}$} & \multirow{3}{*}{ allowed } & hdom & 1190.1 & - \\
\hline & & & & & cdom & 510.3 & - \\
\hline & & & & & bal & 1245.4 & - \\
\hline \multirow{3}{*}{2} & \multirow{3}{*}{$\mathrm{DP}$} & \multirow{3}{*}{ zero } & \multirow{3}{*}{$\mathrm{D} / \mathrm{N}$} & \multirow{3}{*}{ not-allowed } & hdom & 1184.9 & -0.4 \\
\hline & & & & & cdom & 517 & 1.3 \\
\hline & & & & & bal & 1260.5 & 1.2 \\
\hline \multirow{3}{*}{3} & \multirow{3}{*}{ NMPC } & \multirow{3}{*}{ non-zero } & \multirow{3}{*}{$\mathrm{D} / \mathrm{N}$} & \multirow{3}{*}{ allowed } & hdom & 1108.4 & -6.9 \\
\hline & & & & & cdom & 485.6 & -4.8 \\
\hline & & & & & bal & 1180 & -5.3 \\
\hline \multirow{3}{*}{4} & \multirow{3}{*}{ NMPC } & \multirow{3}{*}{ zero } & \multirow{3}{*}{ variable } & \multirow{3}{*}{ allowed } & hdom & 1170.8 & -1.65 \\
\hline & & & & & cdom & 512.7 & 0.5 \\
\hline & & & & & bal & 1247.7 & 0.18 \\
\hline \multirow{3}{*}{5} & \multirow{3}{*}{ NMPC } & \multirow{3}{*}{ non-zero } & \multirow{3}{*}{ variable } & \multirow{3}{*}{ allowed } & hdom & 1080.5 & -9.2 \\
\hline & & & & & cdom & 474.8 & -7 \\
\hline & & & & & bal & 1154.3 & -7.3 \\
\hline \multirow{3}{*}{6} & \multirow{3}{*}{$\mathrm{LOC}\left(\mathrm{COP}_{\mathrm{hp}}=3.5\right)$} & \multirow{3}{*}{ zero } & \multirow{3}{*}{$\mathrm{D} / \mathrm{N}$} & \multirow{3}{*}{ allowed } & hdom & 1185.3 & -0.4 \\
\hline & & & & & cdom & 536.9 & 5.2 \\
\hline & & & & & bal & 1265 & 1.6 \\
\hline & & & & & hdom & 1251.1 to 1304.5 & 5.1 to 9.6 \\
\hline 7 & $\mathrm{LOC}\left(\mathrm{COP}_{\mathrm{hp}}=2.5\right.$ to 6$)$ & zero & $\mathrm{D} / \mathrm{N}$ & not allowed & cdom & 514.4 to 528 & 1 to 3.5 \\
\hline & & & & & bal & 1244.4 to 1296.9 & -0.1 to 4.1 \\
\hline & & & & & hdom & 1163.7 to 1216 & -2.2 to 2.2 \\
\hline & & non-zero & $\mathrm{D} / \mathrm{N}$ & allowed & cdom & 510.8 to 529.3 & 0.1 to 3.7 \\
\hline 8 & $\mathrm{LOC}\left(\mathrm{COP}_{\mathrm{hp}}=2.5\right.$ to 6$)$ & & & & bal & 1239.2 to 1292.6 & -0.5 to 3.8 \\
\hline & & & & & hdom & 1137.8 to 1199.7 & -4.4 to 0.8 \\
\hline 9 & $\mathrm{LOC}\left(\mathrm{COP}_{\mathrm{hp}}=2.5\right.$ to 6$)$ & zero & variable & allowed & cdom & 505.1 to 508.6 & -1 to -0.3 \\
\hline & & & & & bal & 1215.7 to 1278.1 & -2.4 to 2.6 \\
\hline & & & & & hdom & 1135.1 to 1197.6 & -4.6 to 0.6 \\
\hline 10 & $\mathrm{LOC}\left(\mathrm{COP}_{\mathrm{hp}}=2.5\right.$ to 6$)$ & non-zero & variable & allowed & cdom & 502.6 to 506.2 & -1.5 to -0.8 \\
\hline & & & & & bal & 1212.7 to 1275.7 & -2.6 to 2.4 \\
\hline
\end{tabular}

1) Without the concern of TBUD in the ground, zero demand violation margins, and $\mathrm{D} / \mathrm{N}$ electricity prices, the energy use minimization results using NMPC were almost not distinguishable from the results of total energy use minimization over the whole period using DP. Moreover, the results of LOCs based on constant hp COP values gave the results close to the DP results. There were minor circulating fluid mean temperature bound violations, which implies that if an LOC is used, lower-upper temperature bounds should be refined a little bit to avoid these violations but still having a HyGCHP system operating almost globally optimal.

2) When zero TBUD in the ground is taken into account, in the case of $\mathrm{D} / \mathrm{N}$ electricity prices and zero demand violation margins, performances of DP and LOC controllers were again close to each other. Circulating fluid mean temperatures become more oscillatory toward the lower-upper bounds to satisfy the zero TBUD in the ground at the end of the year. Finally, the accumulated cost profile trends are not affected considerably, when compared with the results in Section V.

3) When 5\% demand violation margins and/or a variable electricity price profile are considered, there were reductions in the annual cost relative to the base case up to $9.2 \%$. NMPC was better than LOCs in such cases. However, still the performance of LOCs is acceptable.

4) The above trends were observed for the three types of building loads: heating-dominated, cooling-dominated, and thermally balanced cases.

\section{CONCLUSION}

In this paper, a detailed simulation-based analysis for the energy use minimization of HyGCHP systems was carried out through different controller models and control algorithms and under different scenarios. Moreover, all the controllers were tested on a detailed (finite-volume) emulator model. The results can be summarized as follows.

1) The NARX model with three equivalent states was sufficient to represent the prediction dynamics. This model was used in DP where the results were used as reference values for comparison with NMPC and classical LOC.

2) An SS-ROM was constructed from the emulator model through POD to be used in NMPC. The results from NMPC were almost indistinguishable from the corresponding results of DP when zero demand violation margins are assumed, TBUD in the ground is allowed, and $\mathrm{D} / \mathrm{N}$ electricity prices are considered. This observation implies that NMPC of the HyGCHP system based on energy use minimization over the prediction horizon gives the results very close to the results of DP, which is based on energy use minimization considering the whole period. This important observation can be used to include energy use minimization as the cost function in online control systems like NMPC and putting thermal comfort limits as constraints so that the control system will provide thermal comfort in an energy-optimal way.

3) The temperature dynamics of a borehole system was accurately predicted either by an SS-ROM 
(with six states) or by an input-output model (NARX with three equivalent states). Similar low order models are expected to hold for a borefield system.

4) When a 5\% demand violation margin and/or a variable electricity price were considered, the annual costs changed but not too much. MPC is the most optimal control strategy when applicability, different scenarios, and reduction in annual cost are all considered together. The series of LOCs based on constant COP values gave also good results.

5) An immediate extension of this paper is to a borefield system. Moreover, in the design of a DP controller, gridding can also be done based on heating/cooling loads so that the controller will work under any kind of load, which will not require the loads to be known during controller design.

6) The next extension is to do some experiments to testify the theoretical results obtained from the presented analysis.

\section{REFERENCES}

[1] P. Eskilson, "Thermal analysis of heat extraction boreholes," Ph.D. dissertation, Dept. Math. Phys., Univ. Lund, Lund, Sweden, 1987.

[2] S. P. Kavanaugh and K. Rafferty, Ground-Source Heat Pumps-Design of Geothermal Systems for Commercial and Institutional Buildings. Atlanta, GA, USA: ASHRAE, 1997.

[3] M. A. Bernier, "Closed-loop ground-coupled heat pump systems," ASHRAE J., vol. 48, no. 9, pp. 12-18, 2006.

[4] S. Javed, "Thermal modelling and evaluation of borehole heat transfer," Ph.D. dissertation, Dept. Energy Environ., Chalmers Univ. Technology, Göteborg, Sweden, 2010.

[5] M. Esen and T. Yüksel, "Experimental evaluation of using various renewable energy sources for heating a greenhouse," Energy Build., vol. 65, pp. 340-351, Oct. 2013.

[6] H. Esen, M. Inalli, and M. Esen, "Technoeconomic appraisal of a ground source heat pump system for a heating season in eastern Turkey," Energy Convers. Manage., vol. 47, nos. 9-10, pp. 1281-1297, 2006.

[7] H. Esen, M. Inalli, and M. Esen, "A techno-economic comparison of ground-coupled and air-coupled heat pump system for space cooling," Build. Environ., vol. 42, no. 5, pp. 1955-1965, 2007.

[8] A. Arteconi, C. Brandoni, G. Rossi, and F. Polonara, "Experimental evaluation and dynamic simulation of a ground coupled heat pump for a commercial building," Int. J. Energy Res., vol. 37, no. 15, pp. 1971-1980, 2013.

[9] M. LeBreux, M. Lacroix, and G. Lachiver, "Fuzzy and feedforward control of an hybrid thermal energy storage system," Energy Build., vol. 38 , no. 10 , pp. $1149-1155,2006$.

[10] A. D. Chiasson, "Simulation and design of hybrid geothermal heat pump systems," Ph.D. dissertation, Dept. Civil Archit. Eng., Univ. Wyoming, Laramie, WY, USA, 2007.

[11] E. Atam, C. Verhelst, and L. Helsen, "Development of a controloriented model for borehole dynamics for buildings equipped with ground coupled heat pumps," in Proc. BS Conf., Chambéry, France, Aug. 2013, pp. 2052-2059.

[12] J. R. Cullin and J. D. Spitler, "Comparison of simulation-based design procedures for hybrid ground source heat pump systems," in Proc. 8th Int. Conf. Syst. Simulation Build., Liège, Belgium, 2010, pp. 1-15.

[13] S. Hackel and A. Pertzborn, "Effective design and operation of hybrid ground-source heat pumps: Three case studies," Energy Build., vol. 43, no. 12, pp. 3497-3504, 2011.

[14] F. De Ridder, M. Diehl, G. Mulder, J. Desmedt, and J. Van Bael, "An optimal control algorithm for borehole thermal energy storage systems," Energy Build., vol. 43, no. 10, pp. 2918-2925, 2011.

[15] E. Atam, D. Picard, and L. Helsen, "A convex approach to energy use minimization of buildings equipped with hybrid ground-coupled heat pump systems," in Proc. 9th Int. Conf. Syst. Simulation Build. (SSB), Liège, Belgium, Dec. 2014.

[16] E. Atam and L. Helsen, "A convex approach to a class of non-convex building HVAC control problems: Illustration by two case studies," Energy Build., vol. 93, pp. 269-281, Apr. 2015.
[17] C. Verhelst, "Model predictive control of ground coupled heat pump systems for office buildings," Ph.D. dissertation, Dept. Mech. Eng., KU Leuven, Leuven, Belgium, 2012.

[18] EPBD (Energy Performance of Buildings Directive): Directive 2010/31/UE, 2010.

[19] (2014). District Heating Cooling (DHC). [Online]. Available: http://www.dhcplus.eu/wp-content/uploads/2012/05/120529_Vision DHC_final.pdf

[20] M. Wetter, "GenOpt-A generic optimization program," in Proc. 7th Int. IBPSA Conf., Rio de Janeiro, Brazil, Aug. 2001, pp. 601-608.

[21] Y. Gu and D. L. O'Neal, "An analytical solution to transient heat conduction in a composite region with a cylindrical heat source," J. Solar Energy Eng., vol. 117, no. 3, pp. 242-248, 1995.

[22] MATLAB System Identification Toolbox, MathWorks, Natick, MA, USA, 2011.

[23] P. Holmes, J. L. Lumley, and G. Berkooz, Turbulence, Coherent Structures, Dynamical Systems and Symmetry. Cambridge, U.K. Cambridge Univ. Press, 1996.

[24] P. Astrid, "Reduction of process simulation models: A proper orthogonal decomposition approach," Ph.D. dissertation, Dept. Elect. Eng., Technische Univ. Eindhoven, Eindhoven, The Netherlands, 2004.

[25] A Transient Simulation Program, TRNSYS 16 Reference Manual, TRNSYS, Madison, WI, USA, 1998.

[26] D. E. Kirk, Optimal Control Theory: An Introduction. Englewood Cliffs, NJ, USA: Prentice-Hall, 1970.

[27] D. P. Bertsekas, Dynamic Programming and Optimal Control, 2nd ed. Belmont, MA, USA: Athena Scientific, 2000.

[28] European Commission. (2014). Commission Staff Working Document: Energy Prices and Costs Report. [Online]. Available: http://ec.europa.eu/ energy/doc/2030/20140122_swd_prices.pdf

[29] ELIA. (2013). Grid Data. [Online]. Available: http://www.elia.be/nl/ grid-data

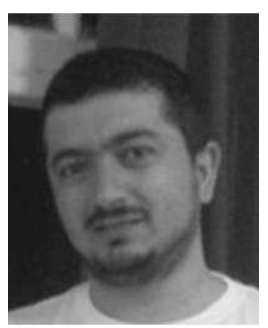

Ercan Atam was born in Elazığ, Turkey, in 1979. He received the B.Sc., M.Sc., and Ph.D. degrees in mechanical engineering from Boğaziçi University, Istanbul, Turkey, in 2002, 2005, and 2010, respectively, with a major in control theory and a minor in optimization.

He was a Teaching Assistant with the Department of Mathematics, Boğaziçi University, during the M.Sc. and Ph.D. periods. He was a Senior Engineer with AVL Turkey, Istanbul, in 2010, and then a Post-Doctoral Researcher with LIMSI-CNRS, Paris, France, from 2010 to 2012, where he was involved in fluid flow control. Since 2012, he has been a Post-Doctoral Researcher in Mechanical Engineering with the Katholieke Universiteit Leuven, Leuven, Belgium, where he is involved in modeling, control, and optimization of energy-efficient buildings.

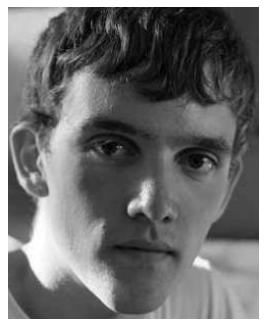

Dieter Patteeuw received the M.S. degree in mechanical engineering from the Katholieke Universiteit Leuven, Leuven, Belgium, in 2011.

$\mathrm{He}$ is currently a Ph.D. Researcher with the Katholieke Universiteit Leuven, more specifically with the Energy Institute, TME Branch (energy conversion), Leuven. 


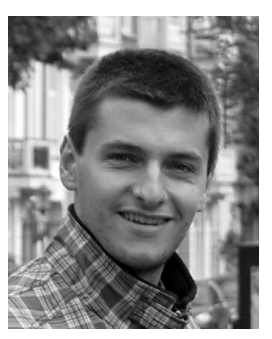

Stefan P. Antonov was born in Sofia, Bulgaria, in 1985. He received the B.Eng. and M.Eng. degrees in automation from the Technical University of Sofia, Sofia, in 2008 and 2010, respectively. He is currently pursuing the Ph.D. degree in mechanical engineering with the Katholieke Universiteit Leuven, Leuven, Belgium.

He was a Bachelor Thesis Student on the ERASMUS program with Linkoping University, Linköping, Sweden, in 2008. From 2009 to 2010, he was a Research and Development Engineer with Visteon Electronics Bulgaria Ltd., Sofia. Since 2010, he has been a Doctoral Researcher with the Department of Mechanical Engineering, Katholieke Universiteit Leuven. His current research interests include ground coupled heat pump systems, model predictive control (MPC), and robustness analysis of MPC.

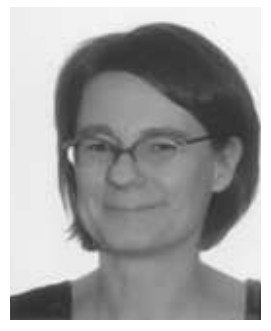

Lieve Helsen was born in Herentals, Belgium, in 1970. She received the master's degree in chemical engineering and the Ph.D. degree in mechanical engineering from the Katholieke Universiteit Leuven, Leuven, Belgium, in 1993 and 2000 , respectively.

She is currently a Professor of Mechanical Engineering, the Program Director of Master Energy Engineering with the Katholieke Universiteit Leuven, and a Core Member of EnergyVille, Thor Park, Waterschei, Belgium. She has authored over 120 publications. Her current research interests include optimal design and control of thermal systems, with a focus on the dynamic behavior of energy systems in buildings, integration of renewable energy sources, and waste-to-energy systems.

Dr. Helsen is a member of the Royal Association of HVAC technology (ATIC), IBPSA-NVL, ODE Vlaanderen, several Flemish (VRWI, FWO, and IWT) and international Expert Panels (SNSF), and Scientific Conference Committees (IBPSA, SSB, and CLIMA). 